Martin Steskal

\title{
Konstruktionszeichnungen zweier Voluten aus dem Prytaneion in Ephesos*
}

\begin{abstract}
Vorbemerkung
Im Zuge der im Sommer 2007 im Prytaneion von Ephesos vorgenommenen Bauaufnahme konnte vom Verfasser an der Südseite des südwestlichen Säulenpostaments (Inv. PR 11/07) des Hestiasaales eine Werkritzung festgestellt werden (Abb. 1-3). Die maximal 0,5 mm in die Marmoroberfläche vertiefte Zeichnung ist lediglich bei entsprechendem Streiflicht mit freiem Auge zu sehen. Da die Oberfläche des Werksteins nach Abschluss der Baumaßnahmen nochmals geglättet worden war, ist die Ritzzeichnung nur noch schwer erkennbar. Die Abnutzung des Werksteins im Laufe der Jahrhunderte sowie die Verwitterung der letzten Jahrzehnte verstärkten diesen negativen Effekt. Dies mag auch der Grund sein, warum der Werkriss seit der Freilegung des Hestiasaales in den Jahren 1955/1956 bis heute unentdeckt blieb1.

Bei den Postamenten samt ihren im Querschnitt herzförmigen Säulenschäften (Eck-Doppelhalbsäulen) und Kompositkapitellen handelt es sich um eine Kombination aus original augusteischen und sekundären Einbauten in den Hestiasaal, die aufgrund des Grabungsbefundes ${ }^{2}$ und stilistischer Kriterien in die erste
\end{abstract}

* Mein Dank gilt dem früheren Grabungsleiter von Ephesos, F. Krinzinger, für die Unterstützung meiner Arbeiten am Prytaneion. Für wertvolle Hinweise sei H. Büsing (Bochum) und G. A. Plattner (Wien) gedankt. Die Arbeiten am Prytaneion von Ephesos werden vom Fonds zur Förderung der wissenschaftlichen Forschung (FWF) finanziert (FWF-Projekt: P19257-G02). - Zusätzlich zu den vom Österreichichen Archäologischen Institut empfohlenen Kurzzitaten $<$ http://www.oeai.at/publik/autoren.html> werden hier folgende verwendet:

Büsing 1987 H. Büsing, Vitruvs Volutenrahmen und die System-Voluten, JdI 102, 1987, 303-338.

Büsing - Lehnhoff 1985 H. Büsing - B. Lehnhoff, Volutenkonstruktion am Beispiel der Erechtheion-Osthalle, AntK 28, 1985, 106-119.

Haselberger 1983 L. Haselberger, Bericht über die Arbeit am Jüngeren Apollontempel von Didyma, IstMitt 33, 1983, 90-123.

IvE Inschriften griechischer Städte aus Kleinasien 11, 1 (Bonn 1979) - 17, 4 (Bonn 1984) = Ephesos: IvE Ia-VIII 2.

Loertscher 1989 Th. Loertscher, Voluta constructa. Zu einem kaiserzeitlichen Volutenkonstruktionsmodell aus Nordafrika, AntK 32, 1989, 82-103.

${ }^{1}$ Zu dem Gebäude: F. Miltner, XXI. Vorläufiger Bericht über die Ausgrabungen in Ephesos, ÖJh 43, 1956-1958, Beibl. 27-36; F. Miltner, Ergebnisse der österreichischen Ausgrabungen in Ephesos im Jahre 1956, AnzWien 94, 1957, 23-25; F. Miltner, XXII. Vorläufiger Bericht über die Ausgrabungen in Ephesos, ÖJh 44, 1959, Beibl. 289-312; F. Eichler, Die österreichischen Ausgrabungen in Ephesos im Jahre 1960, AnzWien 98, 1961, 66-69; F. Eichler, Die österreichischen Ausgrabungen in Ephesos im Jahre 1961, AnzWien 99, 1962, 38-40; F. Eichler, Die österreichischen Ausgrabungen in Ephesos im Jahre 1962, AnzWien 100, 1963, 46; F. Eichler, Die österreichischen Ausgrabungen in Ephesos im Jahre 1963, AnzWien 101, 1964, 40-41; W. Alzinger, Das Regierungsviertel, ÖJh 50, 1972-1975, Beibl. 233-249; W. Alzinger, Augusteische Architektur in Ephesos, SoSchrÖAI 16 (Wien 1974) 51-55; S. G. Miller, The Prytaneion, its Function and Architectural Form (Berkeley 1978) 98-109; D. Knibbe, Der Staatsmarkt. Die Inschriften des Prytaneions, FiE 9, 1, 1 (Wien 1981); H. Thür, Wie römisch ist der sog. Staatsmarkt in Ephesos?, in: M. Meyer (Hrsg.), Neue Zeiten - Neue Sitten. Zu Rezeption und Integration römischen und italischen Kulturguts in Kleinasien. Akten des Internationalen Kolloquiums in Wien 2005, WForsch 12 (Wien 2007) 77-90; A. Bammer, Zur Dekonstruktion römischer Architektur. Studien zur Architektur im Nordbereich der sog. Oberen Agora von Ephesos, Anatolia Antiqua 16, 2008, 165-180.

${ }^{2}$ Nach erster Durchsicht des Fundmaterials von Schnitt 5/07 entlang der Ostwand des Hestiasaales durch S. Ladstätter, der herzlich gedankt sei. Der Zeitpunkt der Errichtung der Unterkonstruktionen der Sitzbänke im Hestiasaal, die die Herzsäulen 


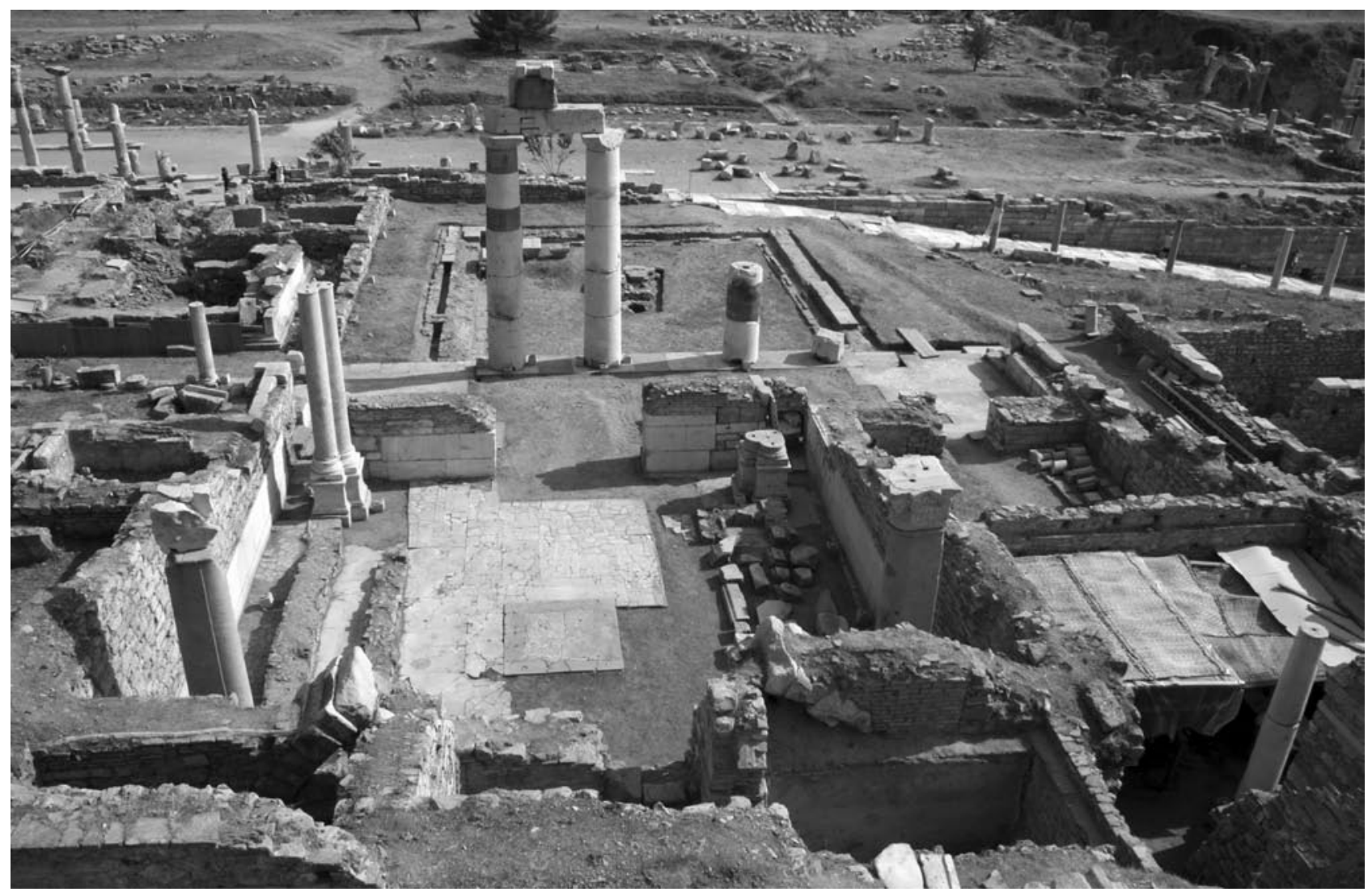

1 Prytaneion von Ephesos - Hestiasaal. Blick nach Süden

Hälfte des 3. Jahrhunderts n. Chr. zu datieren sind ${ }^{3}$. Bei der Konstruktion der Säulenpostamente handelt es sich um individuelle Maßanfertigungen, die auf die natürlichen Terrainverhältnisse Rücksicht nehmen. Diese individuellen Lösungen spiegeln sich in den unterschiedlichen Maßen der jeweiligen Bauglieder wider. Die vier Säulensockel aus stark gemasertem, graublauem Marmor stehen jeweils $0,90 \mathrm{~m}$ von den vier Wänden des Hestiasaales entfernt. Als Fundament für alle Sockel diente der anstehende Fels. Die Säulensockel selbst bestehen aus einer Plinthe (H 0,46 m; Ausnahme: Nordwestsäule: H 0,38 m) und einem 0,89-0,90 m hohen, mit Deck- und Fußprofil versehenen Sockel (Inv. PR 11/07. 13/07. 16/07. 20/07). An der Sockelplinthe der Südwestsäule (Inv. PR 11/07) ist am oberen Rand eine Werkbosse stehengeblieben. Die einzelnen Sockel und Plinthen sind analog zu den Säulen an der Innenseite verkröpft. Darüber befindet sich je eine attische Basis mit angearbeiteter Plinthe (Inv. PR 12/07. 14/07. 17/07. 21/07). Der nordwestliche Säulenschaft wurde vom Ausgräber F. Miltner 1955 noch in situ angetroffen (Inv. PR 22/07). Der südöstliche (Inv. PR 15/07) und der nordöstliche (Inv. PR 18/07) Säulenschaft wurden in Sturzlage aufgefunden und 1956 wieder aufgestellt; der südwestliche Schaft wurde nicht mehr angetroffen. Die Höhe der Säulenschäfte ist durchweg unterschiedlich: H Südostsäule: 4,12 m; H Nordwestsäule: 4,16 m; H Nordostsäule: 4,23 m. Während die beiden nördlichen Säulen aus grauem Granit bestehen, ist die südliche aus graublauem Marmor mit grober weißer Zeichnung gefertigt. In Sturzlage - allerdings im Bereich der Südostsäule - fand sich ein gut erhaltenes, 0,74 m hohes,

baulich bereits berücksichtigen, dient in diesem Kontext als terminus ante quem. Renovierungsarbeiten im Prytaneion-Bezirk durch einen gewissen M. Aurelius Artemidorus Metrodorianus kurz vor der Mitte des 3. Jhs. n. Chr. sind zudem inschriftlich nachgewiesen: IvE 3071.

${ }^{3}$ Dies betrifft insbesondere die attischen Basen mit angearbeiteter Plinthe sowie die im Querschnitt herzförmigen Kompositkapitelle. Letztere setzen sich qualitativ von älteren ephesischen Exemplaren deutlich ab. Vgl. dazu die severischen Kompositkapitelle vom südlichen Hafentor; G. A. Plattner, Ephesische Kapitelle des 1. und 2. Jhs. n. Chr. Form und Funktion kaiserzeitlicher Architekturdekoration in Kleinasien (Diss. Universität Wien 2003) 127-129. - Für Hinweise zur Datierung sei G. A. Plattner gedankt. 
im Querschnitt herzförmiges Kompositkapitell aus weißem Marmor, das im Jahr 1956 auf die nordwestliche Säule gesetzt wurde (Inv. PR 23/07). Fragmente eines weiteren formgleichen Kapitells wurden von F. Miltner aus dem Versturzmaterial geborgen, zusammengesetzt und 1961 auf die Nordostsäule gesetzt (Inv. PR 19/07. 42/07). Das Fragment eines dritten formgleichen Kapitells konnte unter den von den Ausgräbern im Hestiasaal aufgelegten Baugliedern festgestellt werden (Inv. PR 28/07).

Der in den letzten Jahrzehnten ständig gewachsene Kenntnisstand zu antiken Konstruktionszeichnungen kann nunmehr um die Werkritzung zweier Voluten aus dem Prytaneion in Ephesos erweitert werden. Trotz der zahlreichen Bauzeichnungen, die beispielsweise in Rom ${ }^{4}$, Didyma ${ }^{5}$, Priene $^{6}$, Sardes ${ }^{7}$, Pergamon $^{8}$, Aphrodisias ${ }^{9}$, aber auch in Ephesos ${ }^{10}$ vorgefunden worden waren, fehlten mit Ausnahme einer Ritzzeichnung aus dem kaiserzeitlichen Thysdrus (Tunesien) ${ }^{11}$ bislang Werkrisse

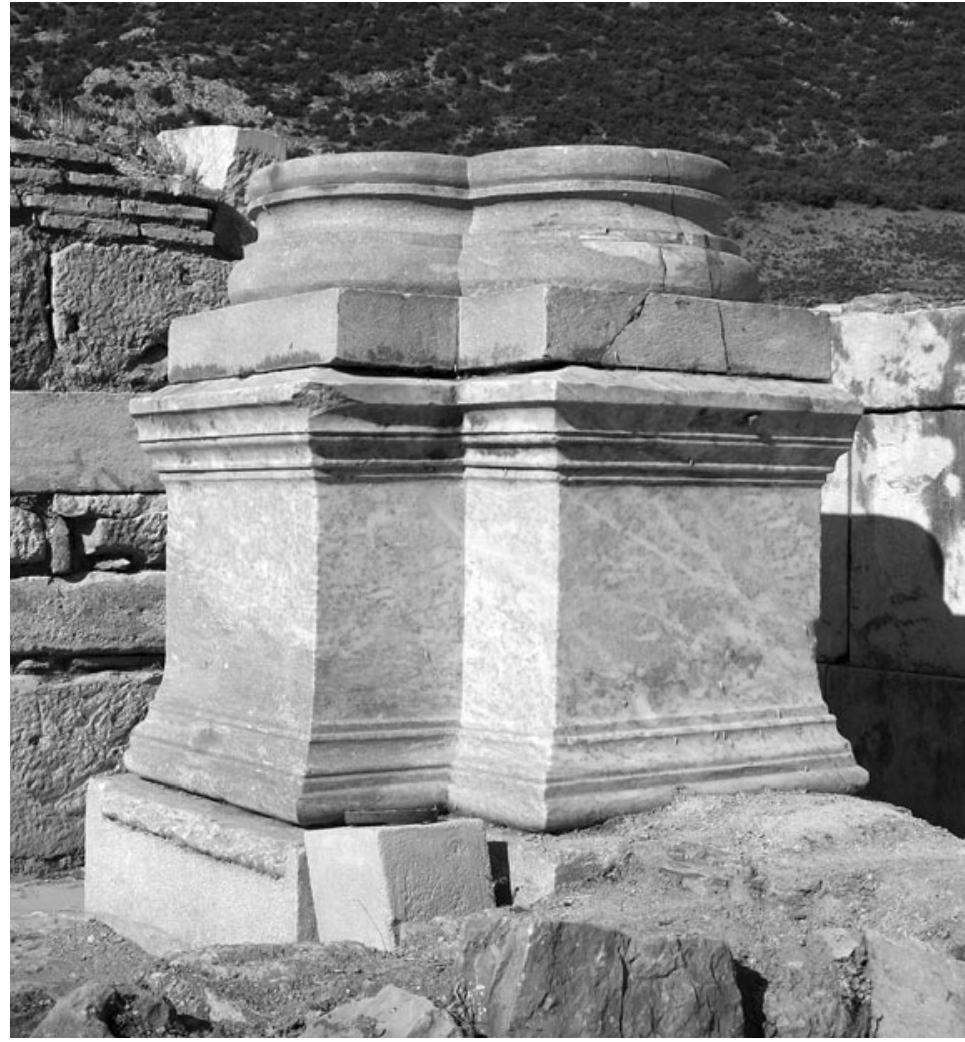

2 Prytaneion - Hestiasaal. Südwestliches Säulenpostament von Voluten. So wurden selbst unter den zahlreichen Zeichnungen am Jüngeren Apollotempel von Didyma keine Voluten oder Kapitelle, sondern lediglich Konstruktionslinien und Einstichpunkte in den Volutenaugen bereits fertiggestellter Kapitelle vorgefunden ${ }^{12}$. Komplexe dreidimensionale Architekturglieder wie Kapitelle ließen sich offenbar nur schwer auf zweidimensionale Zeichnungen übertragen - um ihre Plastizität und Proportionen dem Steinmetzen besser wiedergeben zu können, wurden offenbar Modelle aus Stein oder Holz angefertigt ${ }^{13}$.

${ }^{4}$ L. Haselberger, Ein Giebelriß der Vorhalle des Pantheon. Die Werkrisse vor dem Augustusmausoleum, RM 101, 1994, 279-308;

G. Jenewein, Musterzeichnung einer Basis auf einem Gesimsblock der Basilica Ulpia, RömHistMitt 48, 2006, 69-85.

${ }^{5}$ L. Haselberger, Werkzeichnungen am jüngeren Didymeion, IstMitt 30, 1980, 191-215; Haselberger 1983, 82-103; L. Haselberger, Die Werkzeichnung des Naiskos im Apollontempel von Didyma, in: Bauplanung und Bautheorie der Antike. Bericht über ein Kolloquium in Berlin vom 16. 11.-18. 11. 1983, DiskAB 4 (Berlin 1984) 111-119; L. Haselberger, Aspekte der Bauzeichnungen von Didyma, RA 1991, 99-113.

${ }^{6}$ W. Koenigs, Der Athenatempel von Priene. Bericht über die 1977-82 durchgeführten Untersuchungen, IstMitt 33, 1983, 165-168; W. Koenigs, Pytheos, eine mythische Figur in der antiken Baugeschichte, in: Bauplanung und Bautheorie der Antike (Anm. 5) 89-94 zur sog. Pytheosskizze, die das Gebälk des Athenatempels von Priene im Maßstab $1: 48$ grob wiedergibt. 7 Haselberger 1983, 121.

8 s. R. Bohn, Die Theaterterrasse, AvP 4 (Berlin 1896) 53 Taf. 36.

${ }_{9}$ Haselberger 1983, $121 \mathrm{f}$. Anm. 111.

${ }^{10}$ U. Schädler, Griechische Geometrie im Artemision von Ephesos, in: U. Muss (Hrsg.), Der Kosmos der Artemis von Ephesos, SoSchrÖAI 36 (Wien 2001) 279-287.

${ }^{11}$ Vorgelegt von Loertscher 1989, 82-103; s. auch J. P. Heisel, Antike Bauzeichnungen (Darmstadt 1993) 214-216; L. Haselberger, Architectural Likenesses: Models and Plans of Architecture, JRA 10, 1997, $91 \mathrm{f}$.

12 s. Haselberger 1983, 97 f. Taf. 16; s. auch R. Martin, Chapiteaux ioniques de l'Asclépieion d'Athènes, BCH 68/69, 1944/1945, 353 mit Anm. 1; G. P. Stevens, The Volute of the Capital of the Temple of Athena at Priene, MemAmAc 9, 1931, 136 mit Anm. 2.

${ }^{13}$ Haselberger 1983, 97 f.; Loertscher 1989, 83; J. J. Coulton, Greek Architects and the Transmission of Design, in: Architecture et société de l'archaïsme grec à la fin de la république romaine. Actes du Colloque international organisé par le Centre national de la recherche scientifique et l'Ecole française de Rome, Rome 2-4 décembre 1980, CEFR 66 (Rom 1983 ) 455 f.; F. Tomasello, Un prototipo di capitello corinzio in Sabratha, QuadALibya 13, 1983, 87-103; Th. E. Kalpaxis, Hemiteles. Akzidentelle Unfertigkeit und >Bossen-Stik in der griechischen Baukunst (Mainz 1986) $14 \mathrm{f}$. 


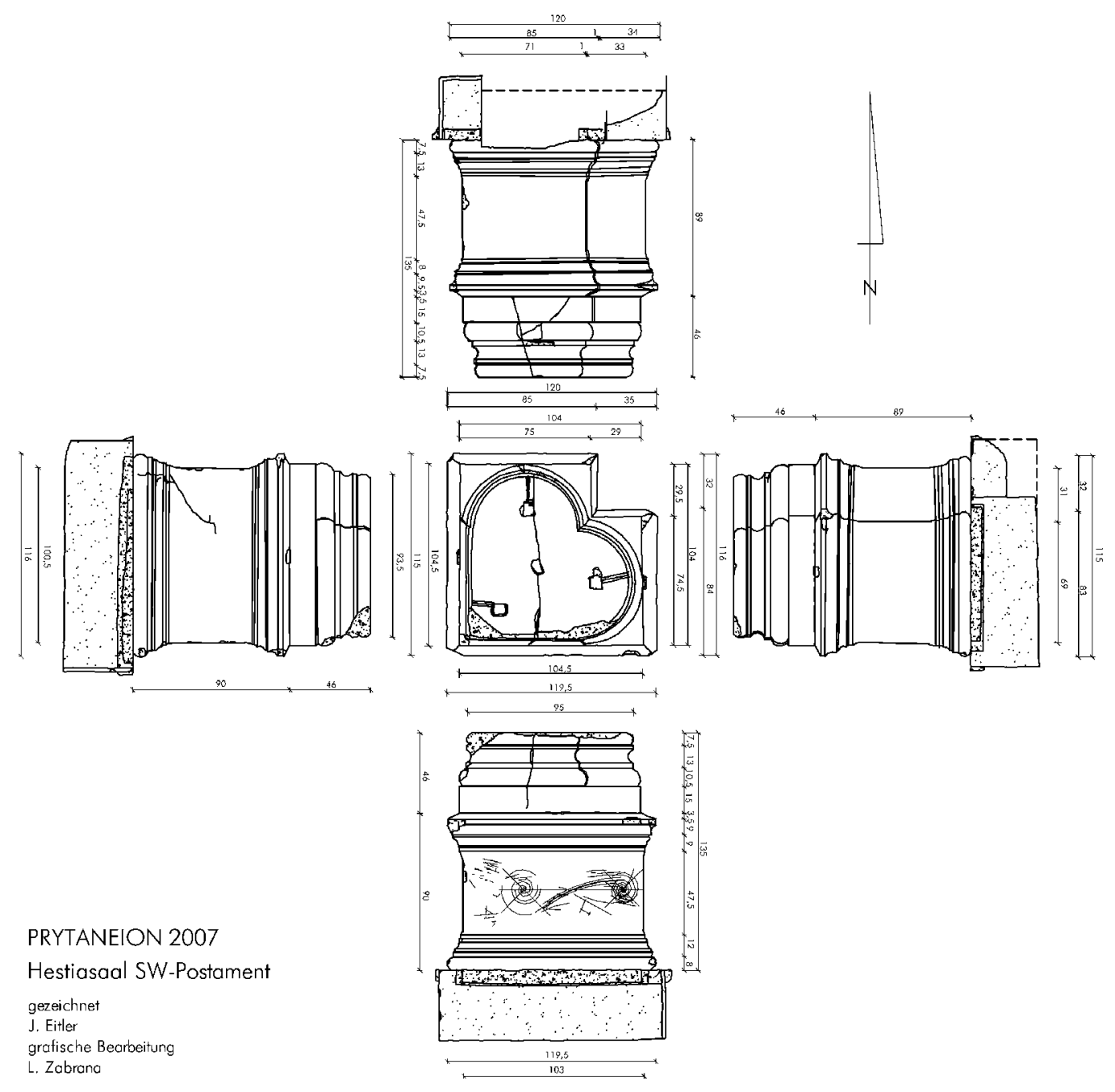

3 Prytaneion-Hestiasaal. Südwestliches Säulenpostament-M. $1: 50$ 


\section{Idealtypische Voluten bei Vitruv}

Die Konstruktion von Voluten in der Antike ist nur bei Vitruv überliefert. Die von ihm beschriebene Volute des idealtypischen ionischen Normalkapitells ${ }^{14}$ unterliegt in diesem Kontext klar definierten Proportionsverhältnissen und geometrischen Bedingungen, die sich auf Vielfache des Radius des Volutenauges zurückführen lassen ${ }^{15}$. Die Grundprinzipien skizziert Vitruv in wenigen Sätzen, die Proportionen der Volute gibt er dabei in relativen Größen an (partes): Deinde hae lineae dividantur ita, ut quattuor partes et dimidia sub abaco relinquantur. Tunc in eo loco, qui locus dividit quattuor et dimidiam et tres et dimidiam partem centrum oculi conlocetur signeturque ex eo centro rotunda circinatio tam magna in diametro, quam una pars ex octo partibus est. Ea erit oculi magnitudine, et in ea catheto respondens diametros agatur. Tunc ab summa sub abaco inceptum, in singulis tetrantorum actionibus dimidiatum oculi spatium minuatur, deinde in eundem tetrantem qui est sub abaco veniat. (Vitr. 3, 5, 6).

Zusammengefasst bedeutet dies (Abb. 4):

- Höhe der Volute: 8 partes (16 r0)

- Durchmesser des Volutenauges: 1 pars (2 r0)

- Höhe der Volute über dem Zentrum des Volutenauges: 41/2 partes (9 r0)

- Höhe der Volute unter dem Zentrum des Volutenauges: 31/2 partes ( 7 r0)

- Die Volute wird durch ein Abschlagen von Viertelkreisen von außen nach innen konstruiert.

- Die Radien der Viertelkreise nehmen mit jedem Viertelkreis um 1/2 pars (1 r0) ab.

Die Textstelle Vitruvs beschreibt jedoch weder, wie viele Viertelkreise oder Windungen nötig sind, um die Volute zu vervollständigen, noch die genaue Position des Volutenauges. Aus den Beschreibungen Vitruvs ist aber abzuleiten: Bliebe die Abnahme der Radien der Viertelkreise um den Radius des Volutenauges konstant, würden acht Viertelkreise genügen, um das Volutenauge zu erreichen.

Folgt man dem vitruvschen Ansatz, lässt sich ein Volutenrahmen rekonstruieren, der durch die ersten vier Viertelkreise der Volutenkurve sowie die Dimension und Position des Volutenauges definiert wird. Wenn nun - nach Vitruv - die Radien der ersten vier Viertelkreise der Volutenkurve jeweils um den Radius des Volutenauges abnehmen, dann beschreiben die Mittelpunkte der Viertelkreise ein Quadrat, dessen Seitenlänge dem Radius des Volutenauges entspricht (Abb. 4). Für jede weitere Volutenwindung ist ein weiteres auf die halbe Seitenlänge reduziertes Quadrat dem jeweils äußeren Quadrat einzuschreiben. Solche Systemvoluten wurden von H. Büsing theoretisch rekonstruiert und ausführlich besprochen ${ }^{16}$ : Von den Einstichpunkten, die zugleich als Mittelpunkte fungieren, werden nacheinander Viertelkreise angerissen. Die Radien der Viertelkreise verkleinern sich dabei von Einstichpunkt zu Einstichpunkt, bis der Zirkelradius dem Radius des Volutenauges entspricht.

${ }^{14}$ Vitr. 3, 5, 5-8. - Zur Diskussion von Volutenkurven s. grundlegend: W. Hoepfner, Zum ionischen Kapitell bei Hermogenes und Vitruv, AM 83, 1968, 213-234; W. Hoepfner, Zwei Ptolemaierbauten. Das Ptolemaierweihgeschenk in Olympia und ein Bauvorhaben in Alexandria, AM Beih. 1 (Berlin 1971) 30-39; Stevens (Anm. 12) 135-144; H. Drerup, Pytheos und Satyros. Die Kapitelle des Athenatempels von Priene und des Maussoleums von Halikarnass, JdI 69, 1954, 1-31; W. Koenigs, Archaische Bauglieder aus Milet: I. Ionische Kapitelle, IstMitt 29, 1979, 187-198; B. Lehnhoff, Das ionische Normalkapitell vom Typus 1:2:3 und die Angaben Vitruvs zum ionischen Kapitell, in: H. Knell - B. Wesenberg (Hrsg.), Vitruv-Kolloquium des Deutschen Archäologen-Verbandes. Darmstadt 17. bis 18. Juni 1982 (Darmstadt 1984) 97-122; Büsing - Lehnhoff 1985, 106-119; Büsing 1987, 305-338; Loertscher 1989, 82-103; O. Bingöl, Vitruvsche Volute am Artemis-Tempel von Hermogenes in Magnesia am Mäander, IstMitt 43, 1993, 399-415; B. F. Weber, Die römischen Heroa von Milet 1, 10 (Berlin 2004) 15-19. - Zum vitruvschen Normalkapitell: O. Bingöl, Das ionische Normalkapitell in hellenistischer und römischer Zeit in Kleinasien, IstMitt Beih. 20 (Tübingen 1980) 132-153; B. Wesenberg, Beiträge zur Rekonstruktion griechischer Architektur nach literarischen Quellen, AM Beih. 9 (Berlin 1983) 132-143.

${ }^{15}$ s. dazu ausführlich Büsing 1987, 305-338. - Die bei Vitruv beschriebene Volute wird seit der Renaissance immer wieder sneu< erfunden; so etwa im 16. Jh. von S. Serlio, Regole generali di architettura sopra le cinque maniere degli edifici (...) con gli esempi delle antichità che, per la magior parte, concordano con la dottrina di Vitruvio (Venedig 1537); G. Salviati, Regola di far perfettamente col compasso la voluta ionica et del capitello ionico et d'ogni altra sorte (Venedig 1552); G. Philandrier, Gulielmi Philandri Castilionii in decem libros M. Vitruvii Pollionis de Arquitectura Annotationes (Rom 1544); im 17. Jh. von N. Goldmann, Tractatus de Stylometris (Leiden 1662); im 19. Jh. von G. Schwarz, Die Schneckenlinie des ionischen Säulenkapitäls, Allgemeine Bauzeitung 1837, 76-82. 88-93; s. auch O. Puchstein, Das ionische Capitell (Berlin 1887).

${ }^{16}$ Büsing 1987, 305-338. 


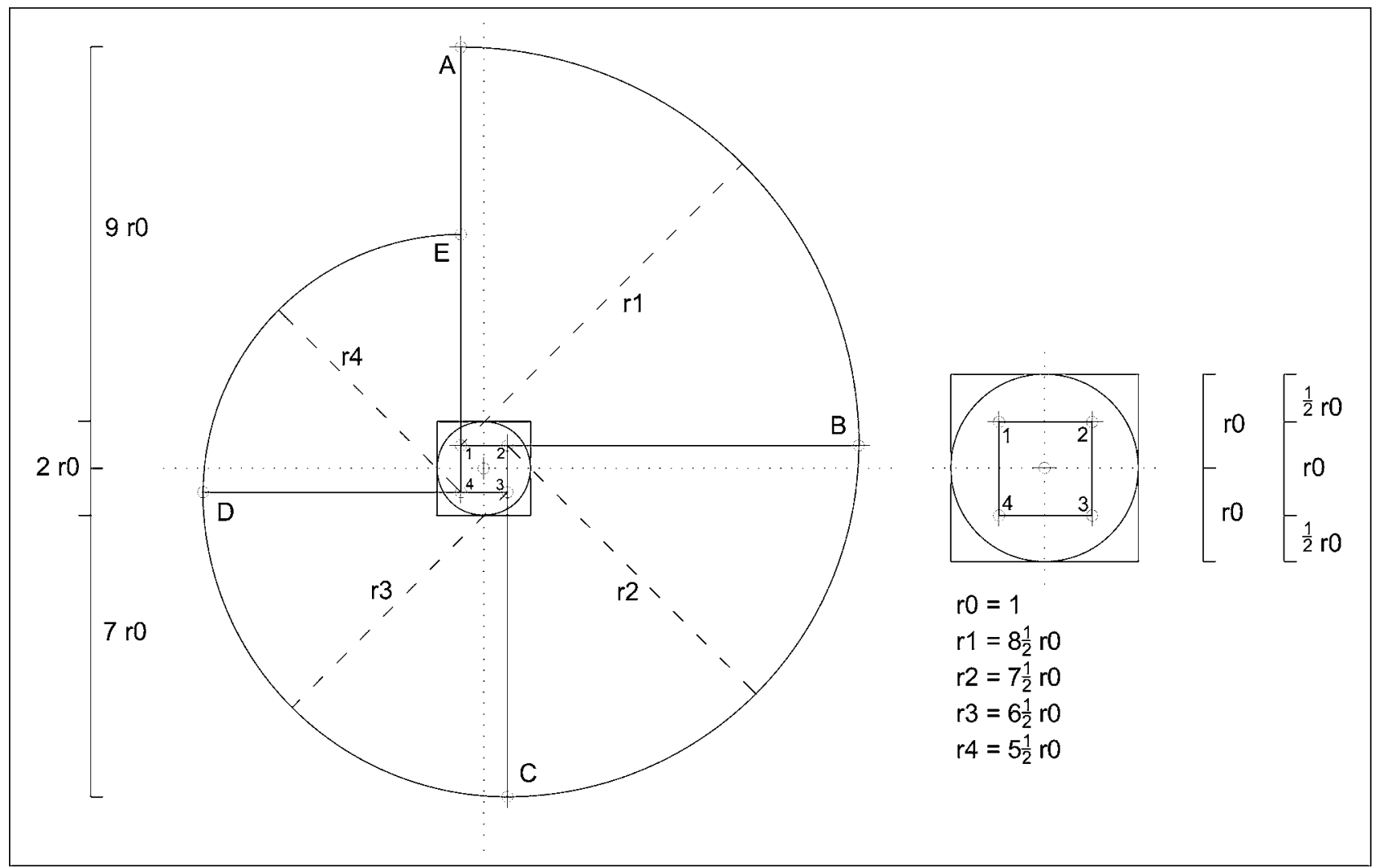

4 Idealtypischer Volutenrahmen nach Vitruv

Um gleich dimensionierte Voluten einer Kapitellserie produzieren zu können, ohne die nötigen Zirkelschläge am Werkstück selbst vornehmen zu müssen, bedurfte es freilich einer einfacheren Methode. Für die praktische Konstruktion von Voluten schlug H. Büsing einen Seil- bzw. »Volutenzirkel« vor ${ }^{17}$. Ihm zufolge bestand er aus einem kleinen Nagelbrett mit Seil und Metalldorn, wobei die Nägel entsprechend den Einstichpunkten der Zirkelspitzen auf den jeweiligen Konstruktionszeichnungen ins Nagelbrett geschlagen wurden. War der Volutenzirkel vorbereitet, ging man wie folgt vor ${ }^{18}$ : »Nun wird zum Anreißen das Ende eines Seils mit einer Schlaufe im Zentrum des Volutenzirkels eingehängt, während am anderen Ende in einer zweiten Schlaufe ein Metalldorn eingehängt wird, dessen Spitze bei straffem Seil genau so weit vom Augenzentrum entfernt ist wie ... Punkt A vom Zentrum der Volute [Abb. 4]. Wird nun der Anriss vorgenommen, so bleibt das Seil zunächst an Nagel 1, dann an Nagel 2 usw. hängen; es wird von den dort aufragenden Stiften verkürzt, bis es schließlich den Kreis des Volutenauges berührt. Dieser Kreis wird vorher oder nachher mit dem starren Zirkel angerissen.« Die Verwendung solcher Volutenzirkel ist freilich nicht gesichert ${ }^{19}$. Dass zumindest partiell auch Schablonen bei der Herstellung von Kapitellen eingesetzt wurden, ist in einer Inschrift in Eleusis um 350 v. Chr. überliefert ${ }^{20}$.

${ }^{17}$ Büsing - Lehnhoff 1985, 106-119.

${ }^{18}$ Büsing - Lehnhoff 1985, 108.

${ }_{19}$ Dazu kritisch Loertscher 1989, 84: »Aus dem Fehlen von Konstruktionsspuren in den Volutenaugen der meisten ionischen Kapitelle kann hypothetisch auf Verfahren geschlossen werden, die ein segmentweises Auftragen mithilfe eines Zirkels überflüssig machten. So ist es denkbar, dass auch hier Schablonen oder, wie bereits vorgeschlagen wurde, Volutenzirkel zur Anwendung gelangt sind, obwohl für beide Übertragungsmöglichkeiten konkrete Beweise fehlen. Der unmittelbare Ausgangspunkt zu ihrer Annahme, das Fehlen von Spuren einer nachkonstruierenden Übertragungsweise nämlich, ist als solcher umstritten und verbietet es, die Anwendung derartiger Übertragungsverfahren als die Regel anzunehmen, ..."

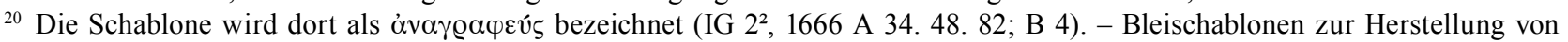
Profilen sind auch materiell erhalten: J. Travlos, ПАРА ЕIГMA, in: Studies in Attic Epigraphy History and Topography Presented to E. Vanderpool, Hesperia Suppl. 19 (Princeton 1982) 172 Taf. 22. 
Von welcher Quelle Vitruv seine Angaben zum ionischen Normalkapitell ableitet, ist umstritten ${ }^{21}$. Nach W. Hoepfner war das Kapitell, von dem Vitruv Konstruktionszeichnungen vorlagen, am von Hermogenes aus Alabanda errichteten Zeus Sosipolis-Tempel in Magnesia verwirklicht ${ }^{22}$. Hermogenes würde demnach neben seinem Hauptwerk, dem magnesischen Artemis Leukophryene-Tempel, auch als ausführender Architekt für die Errichtung des am Ende des 3. Jahrhunderts v. Chr. entstandenen Zeus Sosipolis-Tempels und der diesen umgebenden Agora verantwortlich gezeichnet haben.

Worin lagen nun die Vorteile, einen solchen Werkriss auf einer Oberfläche aus Stein anzufertigen? Das wesentliche Argument für diese Methode lag im glatten und verzugsfreien Zeichengrund ${ }^{23}$. Um den Werkriss deutlich sichtbar zu machen, wurde die Oberfläche des Steins zunächst mit Rötel eingerieben, sodann die Linie eingerissen, die in der Farbe des darunter befindlichen Steins erschien und sich so deutlich abhob. Auf diese Weise konnte auch der Werkriss der Volute auf die geglättete Bosse des Werkstücks übertragen werden. Die Herstellung einer gesamten Serie von Kapitellen mit maßgleichen Voluten ist angesichts des Aufwands allerdings nicht vorstellbar.

\section{Analyse der Werkrisse aus dem Prytaneion}

Die folgende tabellarische Zusammenstellung der Maße und ihre Umrechnung in bekannte römische Einheiten basiert auf dem architektonischen Fußmaß, das hier mit 29,57 cm festgesetzt wird ${ }^{24}$. Da ein digitus $1 / 6$ des pes Romanus ausmacht, ergibt dies für den digitus einen absoluten Wert von 1,85 cm. Sollte dem pes Romanus ein anderer Längenwert entsprechen, so ändert das nichts am Gesamtergebnis, da im Folgenden primär mit moduli, und zwar Vielfachen des Radius des Volutenauges, gerechnet wird ${ }^{25}$. Das Modulsystem bietet sich vor allem auch deshalb an, da sich kleinere Strecken auf römischen Zollstöcken nicht ablesen ließen ${ }^{26}$. Daher werden nur die Hauptmaße der Voluten in Fuß und digiti umgerechnet.

Die Ritzzeichnung weist teilweise Ungenauigkeiten auf, die durch die Führung des Zirkels bedingt sind; auch waren die Messwerte durch die Abnutzung der Werkritzung nicht immer exakt abzugreifen. Die sich dadurch ergebenden Ungenauigkeiten liegen jedoch im Millimeterbereich und somit inner-

${ }^{21}$ So auch H. Knell, Vitruvs Architekturtheorie. Versuch einer Interpretation ${ }^{2}$ (Darmstadt 1991) 101 f.; zur Diskussion um die Quellen von Vitruv: B. Wesenberg, Beiträge zur Rekonstruktion griechischer Architektur nach literarischen Quellen, AM Beih. 9 (Berlin 1983) 109 mit Anm. 479; als Quelle werden Architekten vom 4. bis in das 1. Jh. v. Chr. genannt, etwa Pytheos (Klassik), Hermogenes aus Alabanda und Hermodor aus Salamis (jeweils Hellenismus) und weitere.

22 W. Hoepfner, Zum ionischen Kapitell bei Hermogenes und Vitruv, AM 83, 1968, 213-234; G. Gruben, Griechische Tempel und Heiligtümer ${ }^{5}$ (München 2001) 424-426.

${ }^{23}$ Zur Herstellung des Werkrisses s. A. Petronotis, Zum Problem der Bauzeichnungen bei den Griechen (Athen 1972) 23-30; Haselberger 1983, 92. 97; W. Müller-Wiener, Griechisches Bauwesen in der Antike (München 1988) 35 f.; Loertscher 1989, 84.

${ }^{24}$ Es handelt sich dabei um den Mittelwert gemäß F. Hultsch, Griechische und römische Metrologie (Berlin 1862) 71-77; ein differenzierteres Fußmaß kann aus den Konstruktionszeichnungen nicht abgeleitet werden. Zur Problematik der Festsetzung des pes Romanus: J. A. de Waele, Der römische Fuß in Pompeji: der Tempel des Juppiter Capitolinus, BABesch 59, 1984, 1-8; zum Fußmaß allgemein: J.-P. Adam, Roman Building. Materials and Techniques ${ }^{2}$ (London 2001) 41.

${ }^{25}$ Modulsysteme vereinfachten auch schon in der Antike die zeichnerische Planung sowie deren bauliche Umsetzung; vgl. Müller-Wiener (Anm. 23) 31 f. - Es ist grundsätzlich zu diskutieren, inwieweit nicht an Stelle des pes Romanus im kaiserzeit-

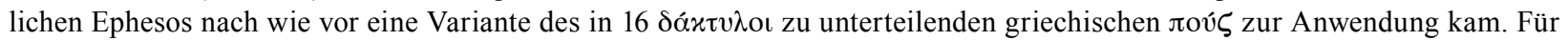
W. Hoepfner, Einführung. Maße - Proportionen - Zeichnungen, in: Bauplanung und Bautheorie der Antike (Anm. 5) 13 f. basiert der pes Romanus, den er mit 29,4 cm bestimmt, auf dem attisch-ionischen Fuß (s. dazu H. Bankel, Zum Fußmaß attischer Bauten des 5. Jahrhunderts v. Chr., AM 98, 1983, 65-99; H. Büsing, Metrologische Beiträge, JdI 97, 1982, 7; E. Buchner, Solarium Augusti und Ara Pacis, RM 83, 1976, 330). Ein entsprechendes Grundmaß kann M. Kadığlu, Die Scaenae frons des Theaters von Nysa am Mäander, Forschungen in Nysa am Mäander 1 (Mainz 2006) 151 nachweisen. H. Thür, Das Hadrianstor in Ephesos, FiE 11, 1 (Wien 1989) 67 berechnete für das ephesische Hadrianstor hingegen ein Grundmaß von $30 \mathrm{~cm}$. Dieses Maß sei - laut H. Thür - nach mündlicher Auskunft von H. Vetters auch an der Domitiansterrasse und in den Hanghäusern 1 und 2 in Ephesos beobachtet worden.

${ }^{26}$ Vgl. H. Büsing, Zur Genauigkeit der Skalen einiger römischer Zollstöcke, KölnJb 24, 1991, 271-285 bes. 283: »Wir glauben, daß die Drittelung des Daktylos und des digitus nicht üblich war. Die Verwendbarkeit und die Genauigkeit der zusammenklappbaren Meßstäbe war also sehr viel geringer als die unserer modernen Zollstöcke.« 


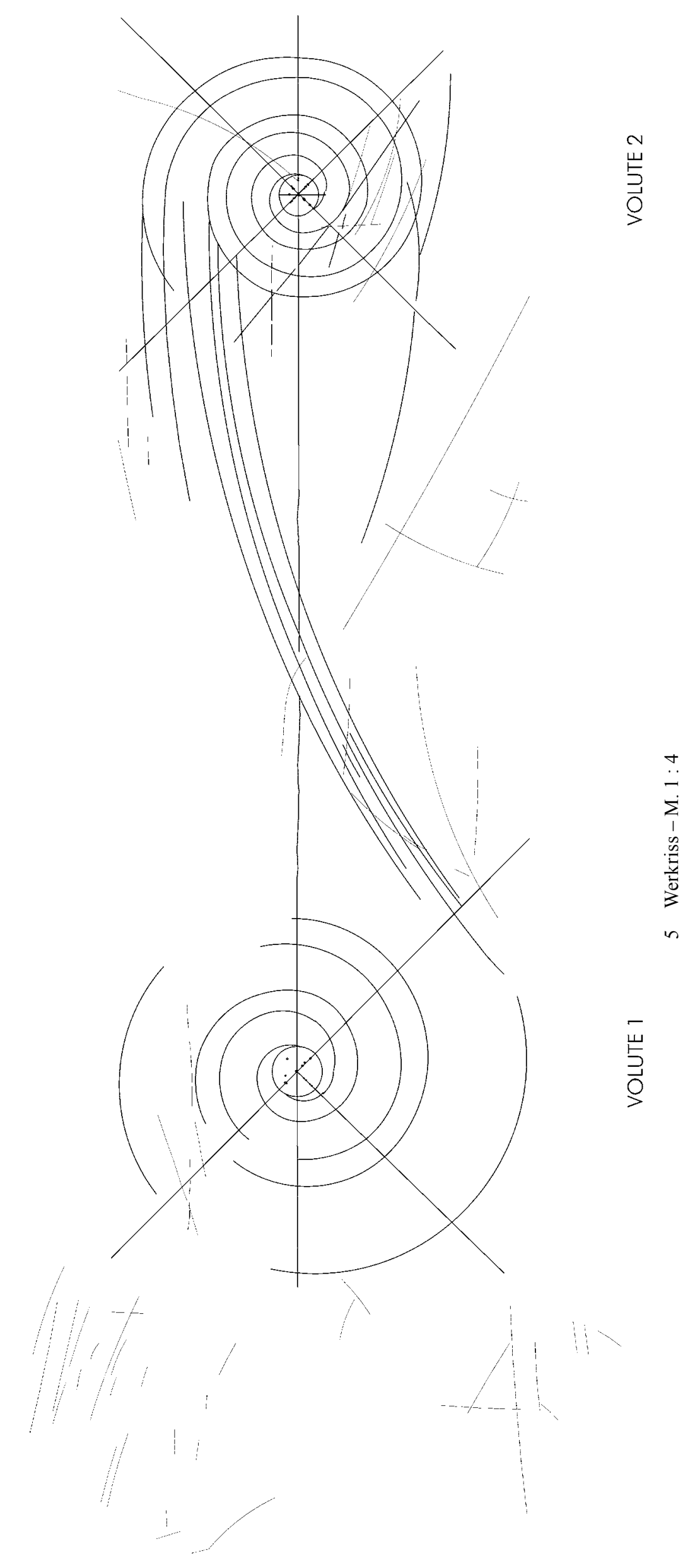


halb der Toleranzgrenze ${ }^{27}$. Da es sich bei einem Kreis per definitionem um eine Figur maximaler Symmetrie handelt, die durch zwei Punkte absolut bestimmt ist, konnten die Viertelkreise mithilfe eines CAD-Programms (AutoCAD ${ }^{\circledR}$ 2008) geometrisch rekonstruiert werden. Bei den Werkrissen handelt es sich wohl um maßstäbliche Zeichnungen (M. $1: 1$ ), was jedoch nicht bewiesen werden $\mathrm{kann}^{28}$. Aufgrund der geglätteten und zugleich abgenutzten Oberfläche sind heute nicht mehr alle Einstichpunkte sichtbar. Sie waren aber geometrisch zu rekonstruieren, wobei eine mögliche ungenaue Führung des Zirkels in diesen Fällen nicht berücksichtigt werden konnte ${ }^{29}$.

\section{Volute 1 (2⿺辶⿸厃𠄌 Windungen)}

Die Ritzzeichnung ist an drei Achsen ausgerichtet, die jeweils als Zentralen durch den Mittelpunkt des Volutenauges geführt werden (Abb. 5. 6). Es handelt

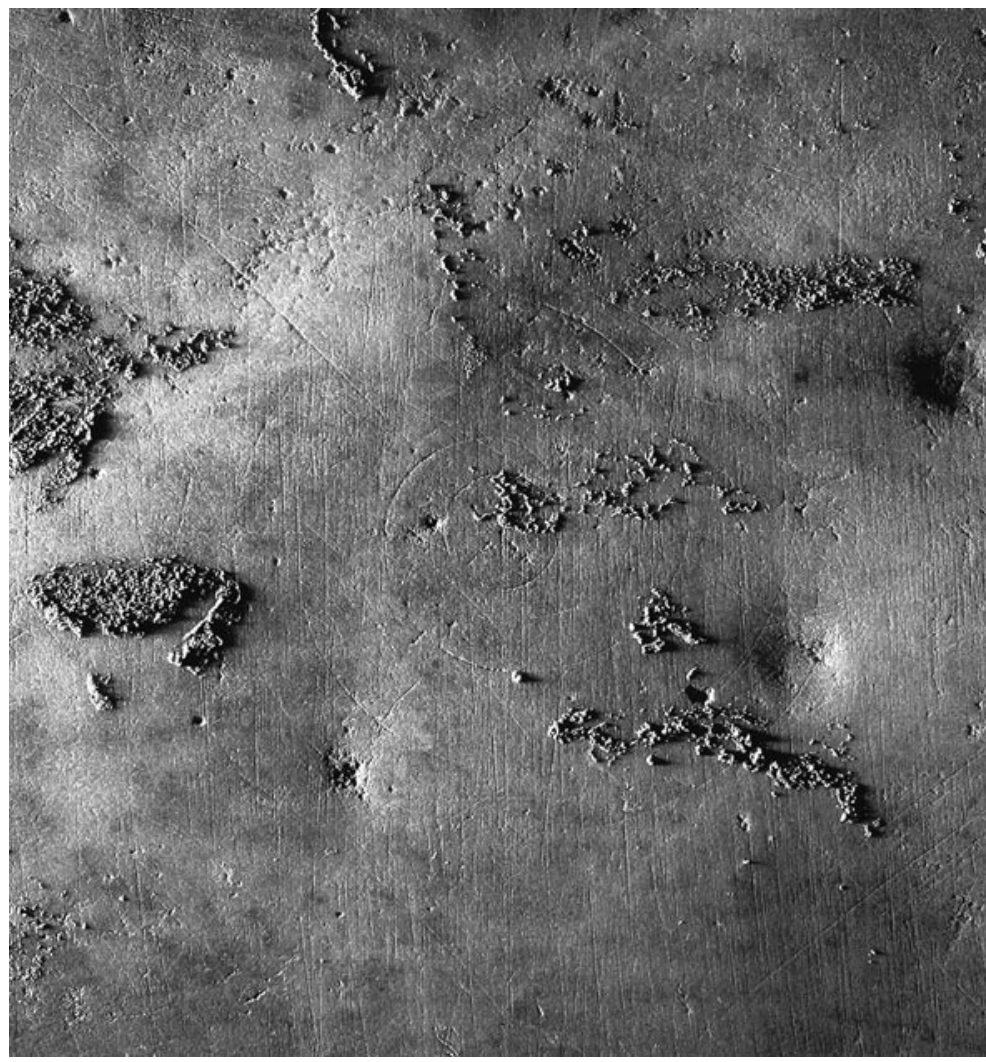

6 Werkriss Volute 1 sich dabei um eine horizontale Konstruktionsachse, die beinahe über die gesamte Südseite des Postaments quer gezogen wurde (L 92,94 cm), sowie zwei um je $45^{\circ}$ aus der Horizontalachse gedrehte Linien, die von links oben nach rechts unten (L $38,05 \mathrm{~cm}$ ) und von links unten nach rechts oben (L 18,54 cm) verlaufen; letztgenannte Linie endet im Zentrum des Volutenauges. Die Konstruktionszeichnung stellt eine stehende Volute mit 21/2 doppelten Windungen dar; durch die doppelten Windungen bildet sich ein Volutensteg. Die äußere Windung berührt nach elf Viertelkreisen das Volutenauge, die innere Windung nach neun. Die Rekonstruktion der Volute ist dank des guten Erhaltungszustandes des Werkrisses bis auf die ersten beiden Viertelkreisbögen der inneren Windung gesichert (Abb. 7-9). Von allen anderen Viertelkreisbögen ist so viel erhalten, dass sie geometrisch zu vervollständigen waren: Die Einstichpunkte des Zirkels orientieren sich an den Zentralen. Die Viertelkreise 4 und 8 der äußeren Windung (Einstichpunkte 7 und 15), die Viertelkreise 4 und 8 der inneren Windung (Einstichpunkte 8 und 16) sowie das Radienpaar $\mathrm{rl} / \mathrm{r} 2$ wurden von demselben Einstichpunkt abgeschlagen (Distanz der Einstichpunkte $<1 \mathrm{~mm}$ ). Auch die Radienpaare r3/r4 und r11/r12 scheinen jeweils vom selben Einstichpunkt aus konstruiert worden zu sein (Distanz der Einstichpunkte $<1,5 \mathrm{~mm}$ ).

\footnotetext{
${ }^{27}$ s. dazu Büsing (Anm. 26) 284: »Unter metrologischen Gesichtspunkten ist die Genauigkeit der römischen Meßwerkzeuge keineswegs als optimal zu bezeichnen. ... Offenbar bestand aber gar kein Bedürfnis für genauere Unterteilungen. Dieses Ergebnis sollte davor warnen, aufgefundene kleine Maße an Bauteilen und anderen Gegenständen zu intensiv zu interpretieren. Die Genauigkeit unserer heutigen, alltäglichen Meßwerkzeuge entspringt nicht der Notwendigkeit des einfachen alltäglichen Lebens, sondern ist erst mit der industriellen und maschinellen Fertigung identischer Elemente ... entstanden. ... Wir können deshalb als Ergebnis festhalten, daß Toleranzen nicht relativ zur gemessenen Strecke, also in Prozent der Ausdehnung einzuschätzen sind, sondern in einem stets gleichen Spielraum von $\pm 2 \mathrm{~mm}$ vom rechnerischen Wert, unabhängig von der Größe der Strecke.«

${ }^{28}$ Die bei weitem größte Zahl antiker Werkrisse ist maßstäblich gezeichnet.

${ }^{29}$ Eine ungenaue Führung des Zirkels ist immer dort zu vermuten, wo das rekonstruierte Einstichloch nicht exakt mit dem auf der Oberfläche vorhandenen Zirkelpunkt übereinstimmt.
} 


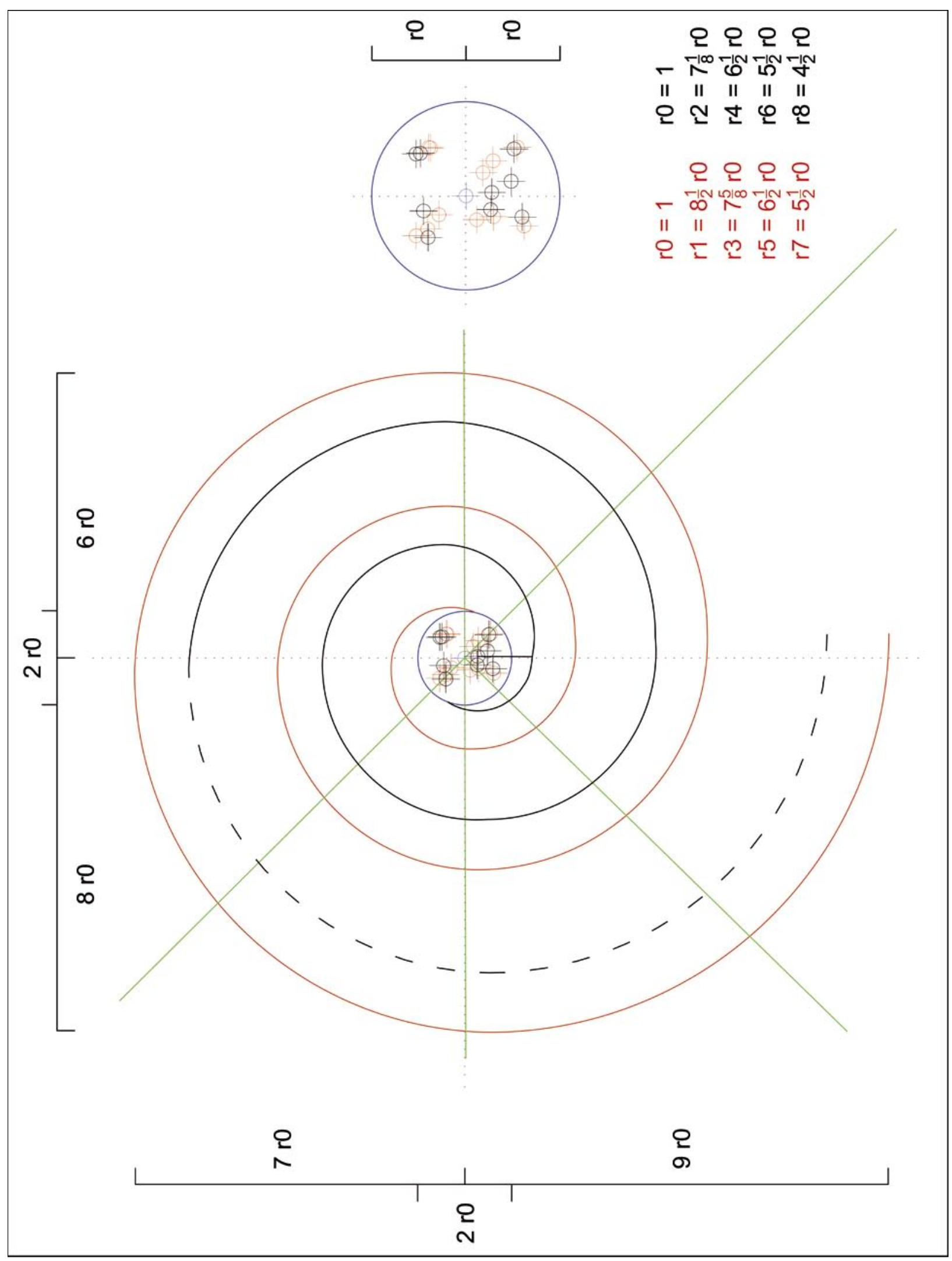

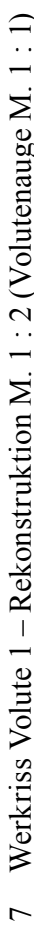




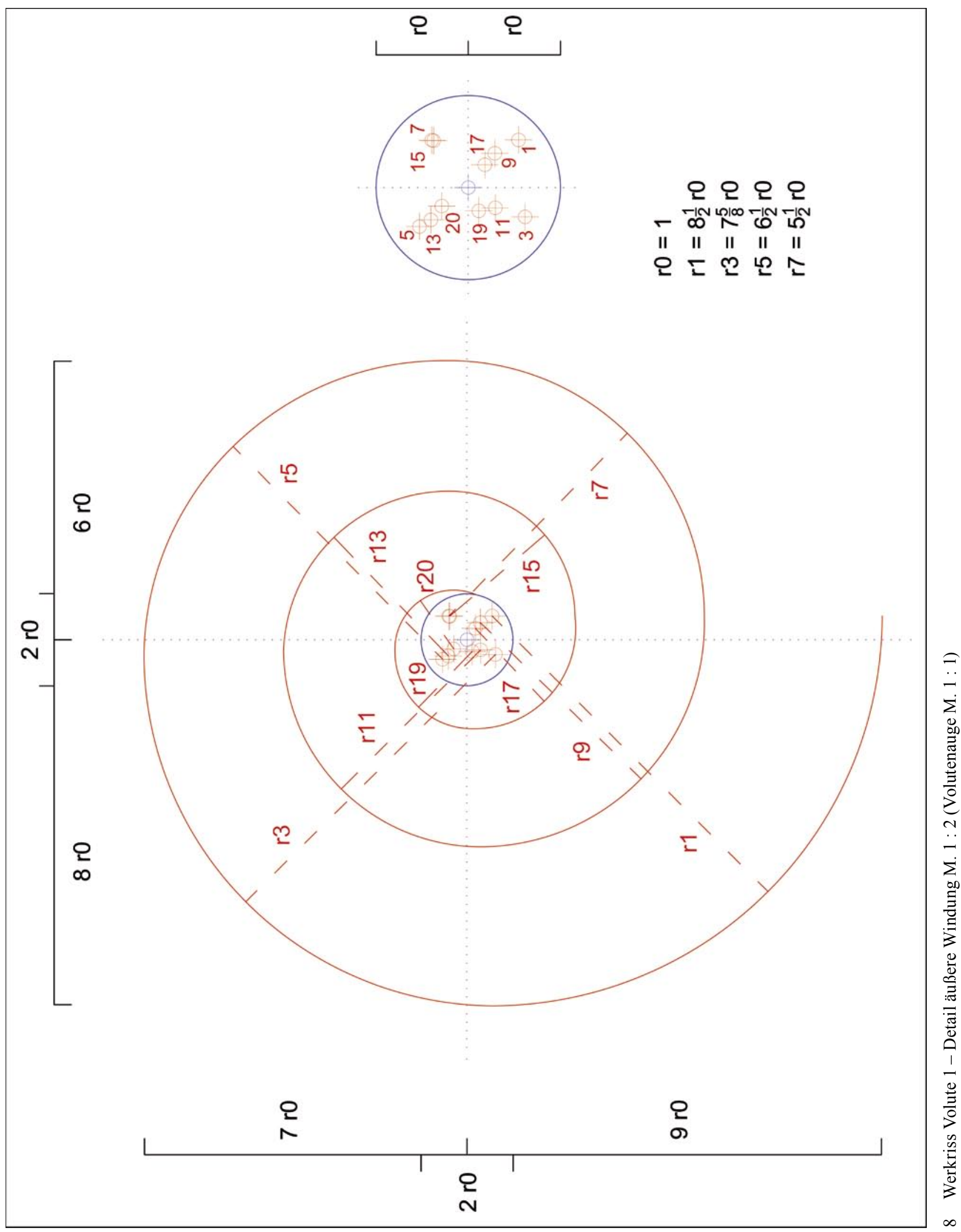




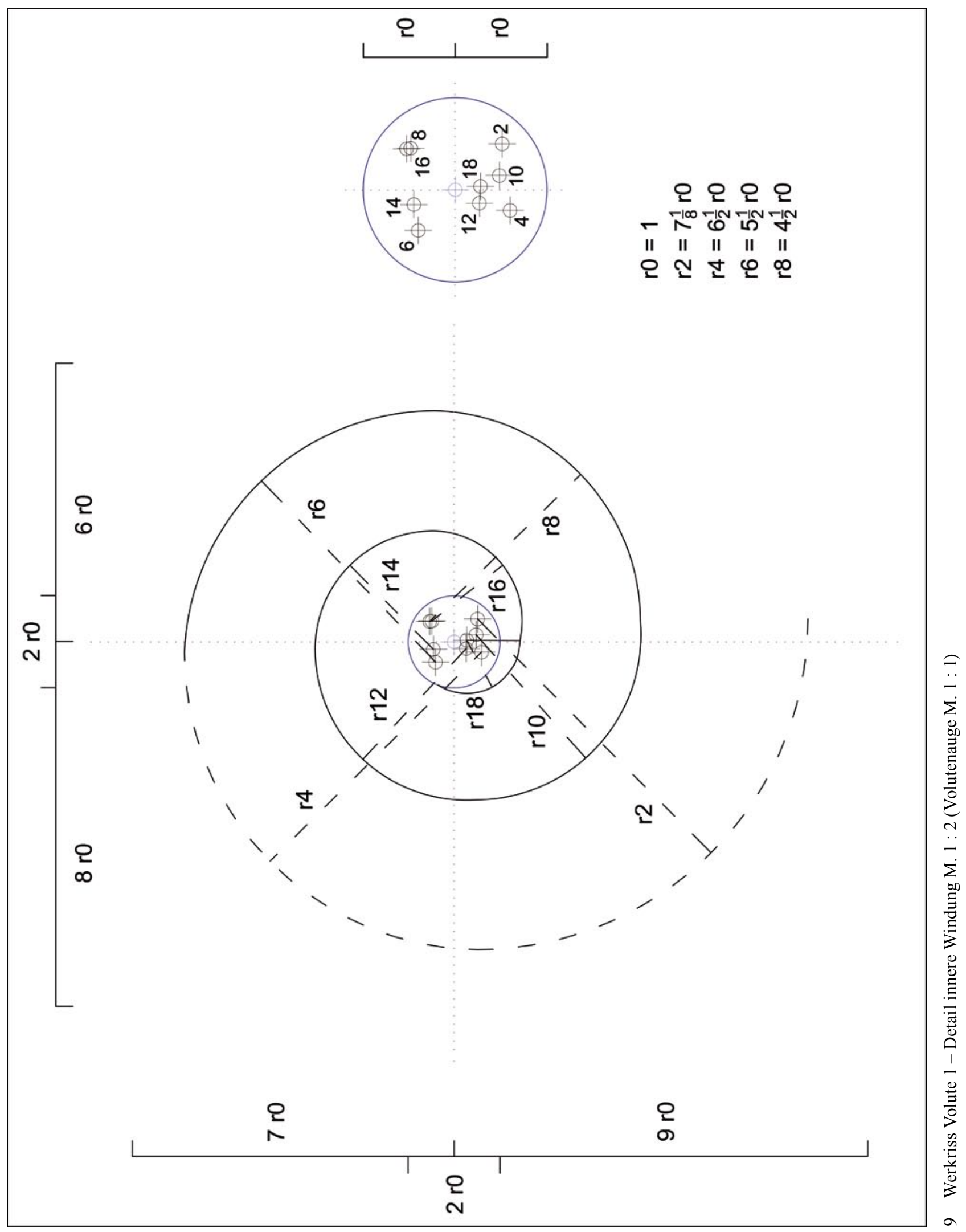


Tabelle 1: Radien Volute $1^{30}$

\begin{tabular}{|c|c|c|}
\hline & Radius & modulus \\
\hline Volutenauge & $1,64 \mathrm{~cm}(\mathrm{r} 0)$ & 1 \\
\hline
\end{tabular}

\begin{tabular}{|c|c|c|c|c|}
\hline Viertelkreis & $\begin{array}{l}\text { Radius äußere } \\
\text { Windung }\end{array}$ & moduli & $\begin{array}{l}\text { Radius innere } \\
\text { Windung }\end{array}$ & moduli \\
\hline 1 & $13,88 \mathrm{~cm}(\mathrm{r} 1)$ & $81 / 2$ & $11,78 \mathrm{~cm}(\mathrm{r} 2)$ & $71 / 8$ \\
\hline 2 & $12,52 \mathrm{~cm}(\mathrm{r} 3)$ & $75 / 8$ & $10,60 \mathrm{~cm} \mathrm{(r4)}$ & $61 / 2$ \\
\hline 3 & $10,64 \mathrm{~cm}(\mathrm{r} 5)$ & $61 / 2$ & $8,97 \mathrm{~cm}(\mathrm{r} 6)$ & $5 \frac{1}{2}$ \\
\hline 4 & $9,10 \mathrm{~cm}(\mathrm{r} 7)$ & $51 / 2$ & $7,51 \mathrm{~cm}(\mathrm{r} 8)$ & $4 \frac{1}{2}$ \\
\hline 5 & 7,98 cm (r9) & $47 / 8$ & $5,88 \mathrm{~cm}(\mathrm{r} 10)$ & 35 \\
\hline 6 & $7,01 \mathrm{~cm}(\mathrm{r} 11)$ & $4 \frac{1}{4}$ & $5,39 \mathrm{~cm}(\mathrm{r} 12)$ & $31 / 4$ \\
\hline 7 & $5,87 \mathrm{~cm}(\mathrm{r} 13)$ & $31 / 2$ & $4,22 \mathrm{~cm}(\mathrm{r} 14)$ & $21 / 2$ \\
\hline 8 & $4,46 \mathrm{~cm}(\mathrm{r} 15)$ & $23 / 4$ & $3,22 \mathrm{~cm}(\mathrm{r} 16)$ & 2 \\
\hline 9 & $3,57 \mathrm{~cm}(\mathrm{r} 17)$ & $21 / 8$ & $1,90 \mathrm{~cm}(\mathrm{r} 18)$ & $1 \frac{1}{8}$ \\
\hline 10 & $2,75 \mathrm{~cm}(\mathrm{r} 19)$ & $15 / 8$ & - & - \\
\hline 11 & $2,10 \mathrm{~cm}(\mathrm{r} 20)$ & $1 \frac{1}{4}$ & - & - \\
\hline
\end{tabular}

Da die Viertelkreise der äußeren und inneren Windungen nicht immer konzentrisch verlaufen, variiert auch die Stärke des Volutensteges. Die Abweichungen werden durch folgende Graphik veranschaulicht ${ }^{31}$ :

Radien Volute 1

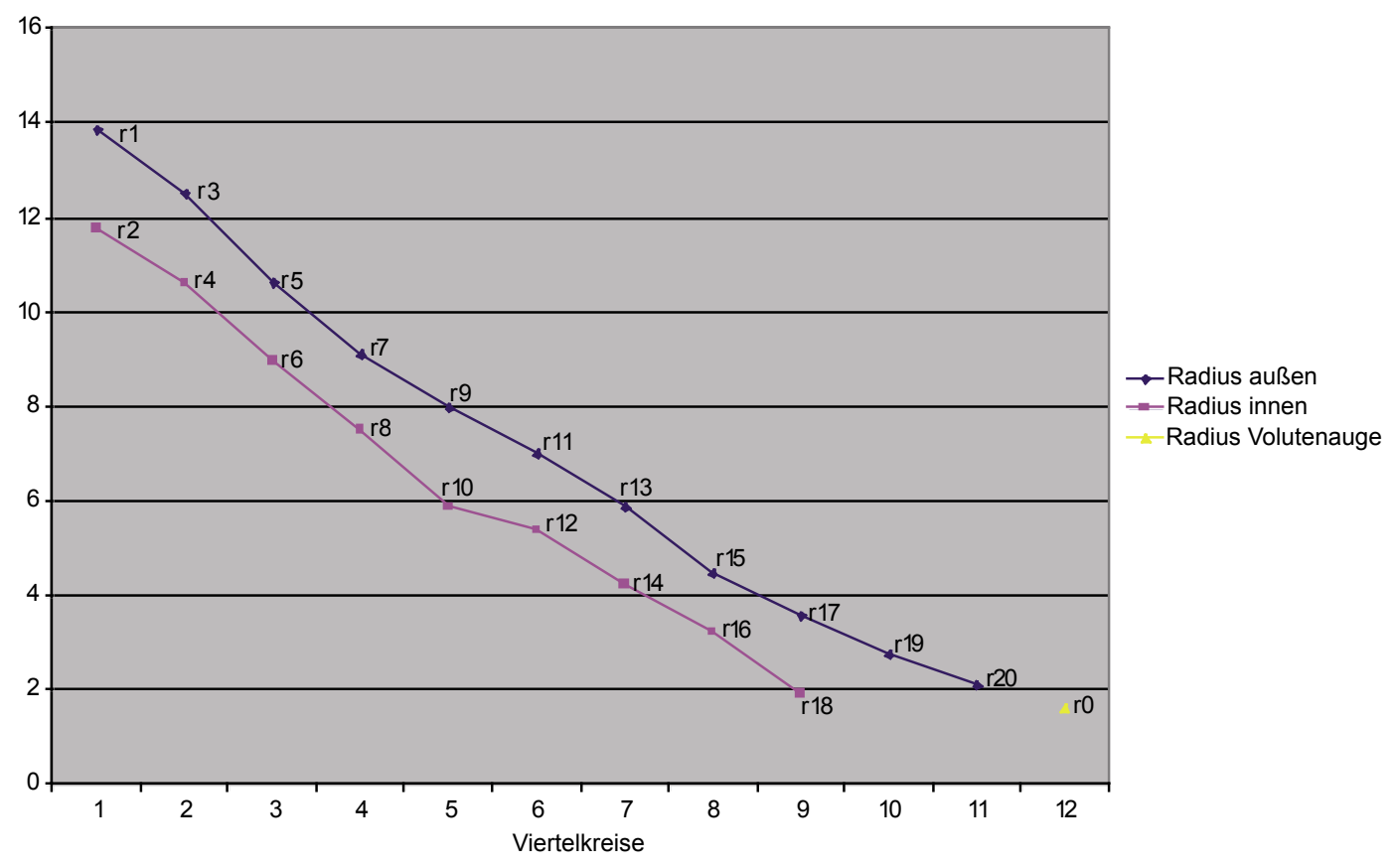

${ }^{30}$ Kursiv dargestellte Werte sind rekonstruiert.

${ }^{31}$ Warum die beiden Windungen des Volutensteges nur teilweise konzentrisch angelegt wurden, ist unverständlich, da dies zu deutlich mehr Einstichpunkten führte. 
Legt man durch das Zentrum des Volutenauges eine horizontale und vertikale Achse und misst an den Schnittpunkten der Achsen mit dem Volutensteg die Breite des Canalis, ist zu sehen, dass sich dieser kontinuierlich verjüngt, die Volute folglich nicht pulsiert. Die Graphik zeigt aber deutlich, dass diese stetige Verjüngung nicht regelmäßig verläuft.

Tabelle 2: Canalis-Breite Volute 1

\begin{tabular}{|c|c|c|}
\hline Schnittpunkt & Breite Canalis & moduli \\
\hline 1 & $4,16 \mathrm{~cm}$ & $2^{1 / 2}$ \\
\hline 2 & $3,57 \mathrm{~cm}$ & $2^{1 / 6}$ \\
\hline 3 & $3,09 \mathrm{~cm}$ & $1^{7 / 8}$ \\
\hline 4 & $2,94 \mathrm{~cm}$ & $1^{4 / 5}$ \\
\hline 5 & $2,82 \mathrm{~cm}$ & $1^{3 / 4}$ \\
\hline 6 & $2,45 \mathrm{~cm}$ & $1^{1 / 2}$ \\
\hline 7 & $2,41 \mathrm{~cm}$ & $1^{1 / 2}$ \\
\hline 8 & $2,15 \mathrm{~cm}$ & $1^{1 / 3}$ \\
\hline 9 & $0,71 \mathrm{~cm}$ & $2 / 5$ \\
\hline 10 & $0,14 \mathrm{~cm}$ & $1 / 10$ \\
\hline
\end{tabular}

Canalis-Breite Volute 1

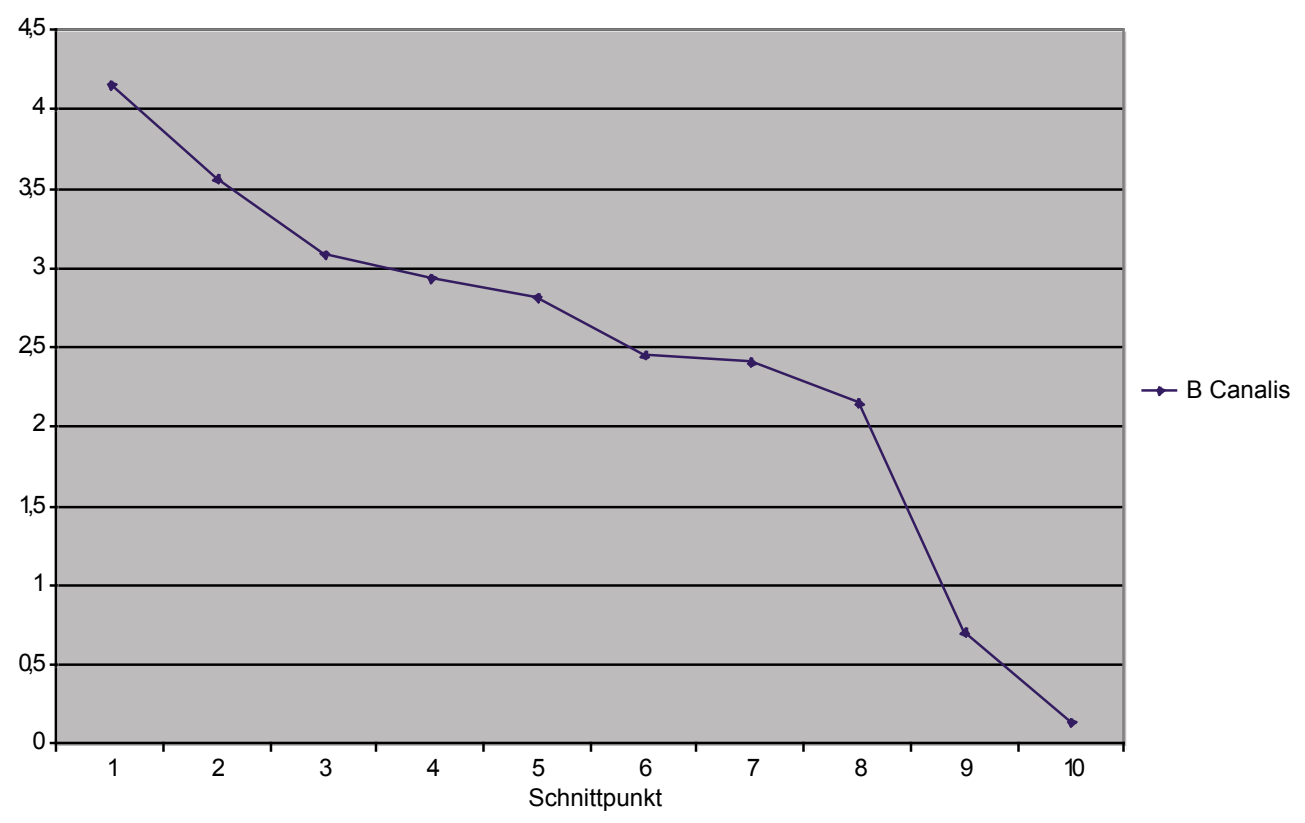

Die Proportionalverhältnisse der Volute orientieren sich an den Vorgaben Vitruvs, stimmen aber nicht in allen Details mit ihnen überein: So verhält sich die Höhe der Volute über dem Zentrum des Volutenauges zu der Höhe darunter tatsächlich wie die geforderten $9 \mathrm{r} 0(14,78 \mathrm{~cm}=8$ digiti $): 7 \mathrm{r} 0(11,49 \mathrm{~cm}=61 / 4$ digiti), folglich $4 \frac{1}{2}: 3 \frac{1}{2}$. Die Gesamthöhe der Volute entspricht den vorgegebenen 8 Durchmessern des Volutenauges $(16 \mathrm{r} 0=26,27 \mathrm{~cm}=141 / 4$ digiti). Die Abnahme der Radien der ersten vier Viertelkreise um den Radius des Volutenauges wird hingegen nur bedingt eingehalten: $8 \frac{1}{2}-75 / 8-61 / 2-51 / 2$. Das maximale Breitenverhältnis der Volute lautet $8 \mathrm{r} 0(13,04 \mathrm{~cm}=7$ digiti $): 6 \mathrm{r} 0(9,95 \mathrm{~cm}=51 / 3$ digiti $)$, somit $4: 3$. Für das Verhältnis Höhe : Breite der Volute ergibt dies $16 \mathrm{r} 0\left(26,27 \mathrm{~cm}=14 \frac{1}{4}\right.$ digiti $): 14 \mathrm{r} 0\left(22,99 \mathrm{~cm}=12^{1 / 2}\right.$ digiti), d. h. $4: 3^{1 / 2}$. 
Tabelle 3: Proportionsverhältnisse Volute 1

\begin{tabular}{|c|c|c|c|}
\hline & $\begin{array}{c}\text { Höhenproportion an } \\
\text { Horizontalachse }\end{array}$ & $\begin{array}{c}\text { Breitenproportion an } \\
\text { Vertikalachse }\end{array}$ & $\begin{array}{c}\text { Proportion } \\
\text { Höhe : Breite }\end{array}$ \\
\hline Volute 1 & $41 \frac{1}{2}: 3^{1 / 2}$ & $4: 3$ & $4: 3 \frac{1}{2}$ \\
\hline
\end{tabular}

Volute $2\left(2 \frac{1}{2}\right.$ Windungen)

Der Werkriss von Volute 2 ist an vier Achsen ausgerichtet, die - wie bei Volute 1 - jeweils als Zentralen durch den Mittelpunkt des Volutenauges geführt werden (Abb. 5. 10). Es handelt sich dabei um die bereits bekannte horizontale Konstruktionsachse (L 92,94 cm), zwei je um $45^{\circ}$ aus der Horizontalachse gedrehte Linien, die von links oben nach rechts unten (L 29,23 cm) und von links unten nach rechts oben (L 30,28 cm) verlaufen, sowie eine vertikale Konstruktionsachse (L 2,99 cm), die das Volutenauge mittig durchmisst und über dieses hinaus nach unten weitergeführt wird. Die Volute besitzt ebenfalls $2 \frac{1}{2}$ doppelte Windungen, wodurch sich auch hier ein Steg bildet. Die äußere Windung berührt nach zehn Viertelkreisen das Volutenauge, die innere Windung nach neun. Im Gegensatz zu der stehenden Volute 1 ist Volute 2 hängend dargestellt. Die Rekonstruktion der Volute ist aufgrund des sehr guten Erhaltungszustandes des Werkrisses gesichert (Abb. 11-13). Von allen Viertelkreisbögen ist so viel erhalten, dass sie geometrisch zu vervollständigen waren. Sieben Radienpaare scheinen jeweils vom selben Einstichpunkt abgeschlagen worden zu sein. Es handelt sich dabei durchweg um konzentrisch angelegte Viertelkreise der äußeren und inneren Windung, und zwar um deren Radienpaare r3/r4, r5/r6, r11/r12, r15/r16 und r17/r18 (Distanz der Einstichpunkte $<1 \mathrm{~mm}$ ) sowie r1/r2 und r9/r10 (Distanz der Einstichpunkte $<1,5 \mathrm{~mm}$ ).

Tabelle 4: Radien Volute 2

\begin{tabular}{|c|c|c|}
\hline & Radius & modulus \\
\hline Volutenauge & $1,28 \mathrm{~cm}(\mathrm{r} 0)$ & 1 \\
\hline
\end{tabular}

\begin{tabular}{|c|c|c|c|c|}
\hline Viertelkreis & $\begin{array}{l}\text { Radius äußere } \\
\text { Windung }\end{array}$ & moduli & $\begin{array}{l}\text { Radius innere } \\
\text { Windung }\end{array}$ & moduli \\
\hline 1 & $9,31 \mathrm{~cm}(\mathrm{r} 1)$ & $71 / 4$ & $8,08 \mathrm{~cm}(\mathrm{r} 2)$ & $61 / 4$ \\
\hline 2 & $7,97 \mathrm{~cm}(\mathrm{r} 3)$ & $61 / 4$ & $6,68 \mathrm{~cm}(\mathrm{r} 4)$ & $51 / 4$ \\
\hline 3 & $7,42 \mathrm{~cm}(\mathrm{r} 5)$ & $53 / 4$ & $6,17 \mathrm{~cm}(\mathrm{r} 6)$ & $47 / 8$ \\
\hline 4 & $6,32 \mathrm{~cm}(\mathrm{r} 7)$ & 5 & $5,20 \mathrm{~cm}(\mathrm{r} 8)$ & 4 \\
\hline 5 & $5,22 \mathrm{~cm}(\mathrm{r} 9)$ & 4 & $4,08 \mathrm{~cm}(\mathrm{r} 10)$ & $31 / 8$ \\
\hline 6 & $4,72 \mathrm{~cm}(\mathrm{r} 11)$ & $3 \frac{3}{4}$ & $3,62 \mathrm{~cm}(\mathrm{r} 12)$ & $27 / 8$ \\
\hline 7 & $3,76 \mathrm{~cm}(\mathrm{r} 13)$ & 3 & $3,09 \mathrm{~cm}$ (r14) & $23 / 8$ \\
\hline 8 & $3,21 \mathrm{~cm}(\mathrm{r} 15)$ & $2 \frac{1}{2}$ & $2,08 \mathrm{~cm}(\mathrm{r} 16)$ & $15 / 8$ \\
\hline 9 & $2,70 \mathrm{~cm} \mathrm{(r17)}$ & $21 / 8$ & $1,55 \mathrm{~cm}(\mathrm{r} 18)$ & $1 \frac{1}{4}$ \\
\hline 10 & $1,94 \mathrm{~cm}(\mathrm{r} 19)$ & $1 \frac{1}{2}$ & - & - \\
\hline
\end{tabular}

Wie bei Volute 1 verlaufen auch die Viertelkreise der äußeren und inneren Windungen von Volute 2 nur teilweise konzentrisch; somit variiert auch hier die Stärke des Volutensteges. Die Abweichungen sind der Graphik 3 (s. S. 387) zu entnehmen.

Legt man auch durch das Zentrum des Volutenauges von Volute 2 eine horizontale und vertikale Achse und misst an den Schnittpunkten der Achsen mit dem Volutensteg die Breite des Canalis, ist zu sehen, dass sich dieser im Wesentlichen kontinuierlich verjüngt. Lediglich zwischen Schnittpunkt 6 und 7 gibt es eine kleine Anomalie, die aber unter $1 \mathrm{~mm}$ liegt. Die Verjüngung verläuft regelmäßiger als bei Volute 1. 


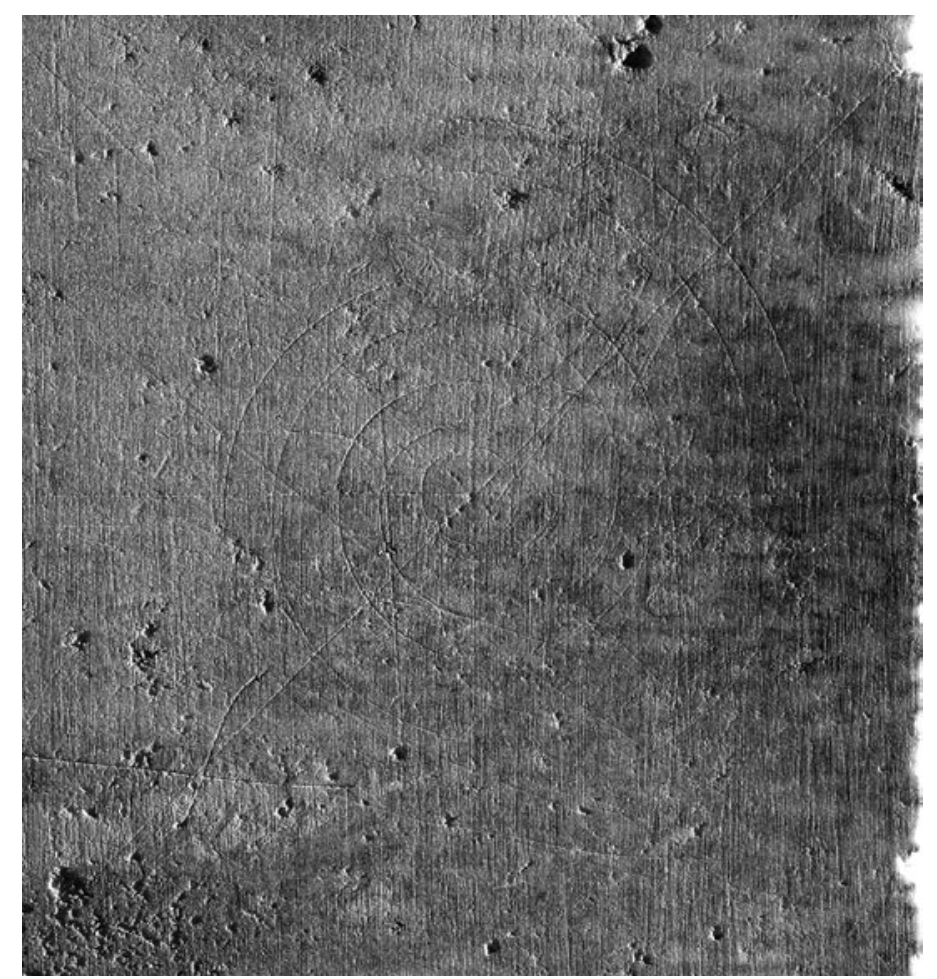

10 Werkriss Volute 2

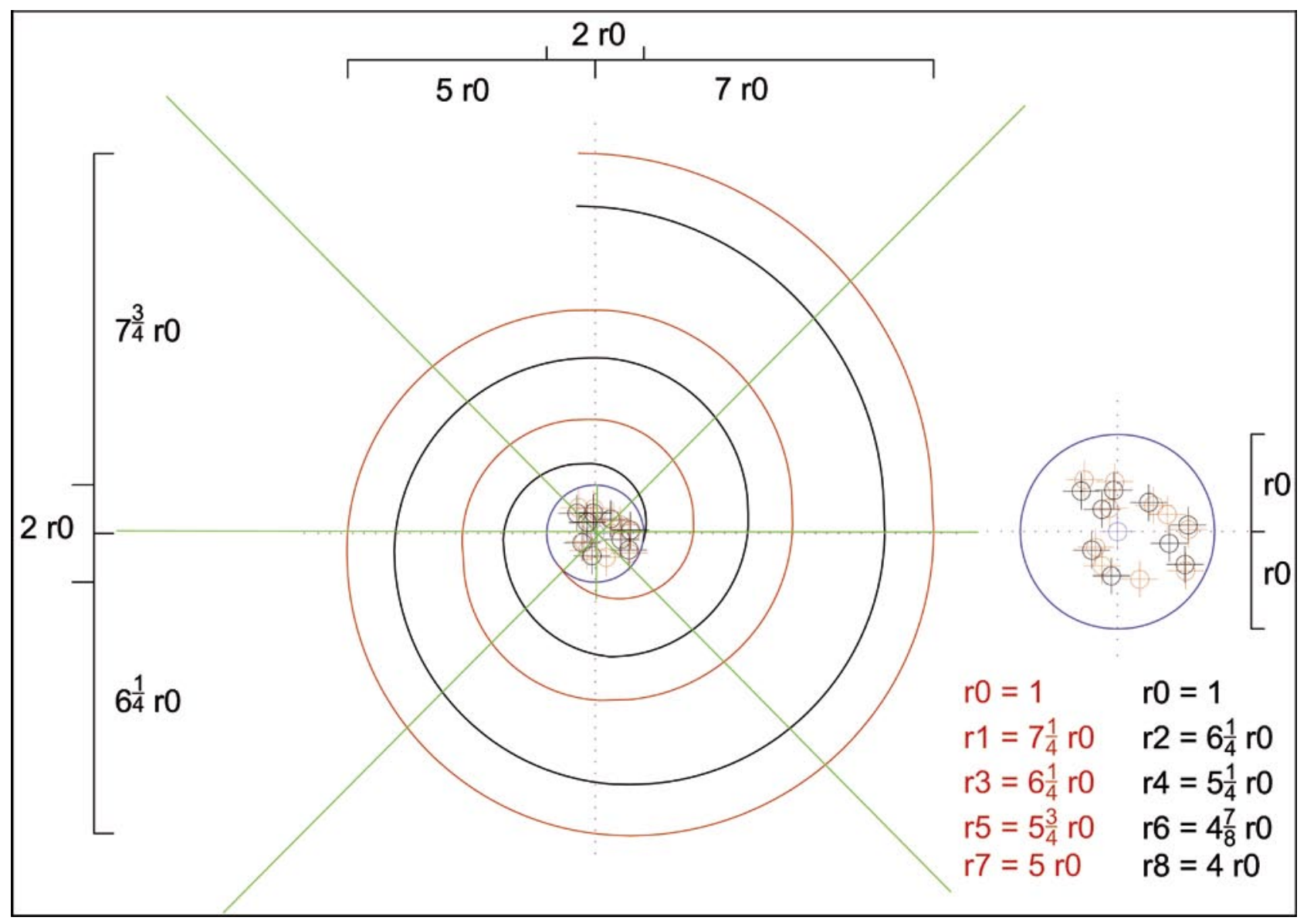

11 Werkriss Volute 2 - Rekonstruktion M. $1: 2$ (Volutenauge M. $1: 1$ ) 
Radien Volute 2

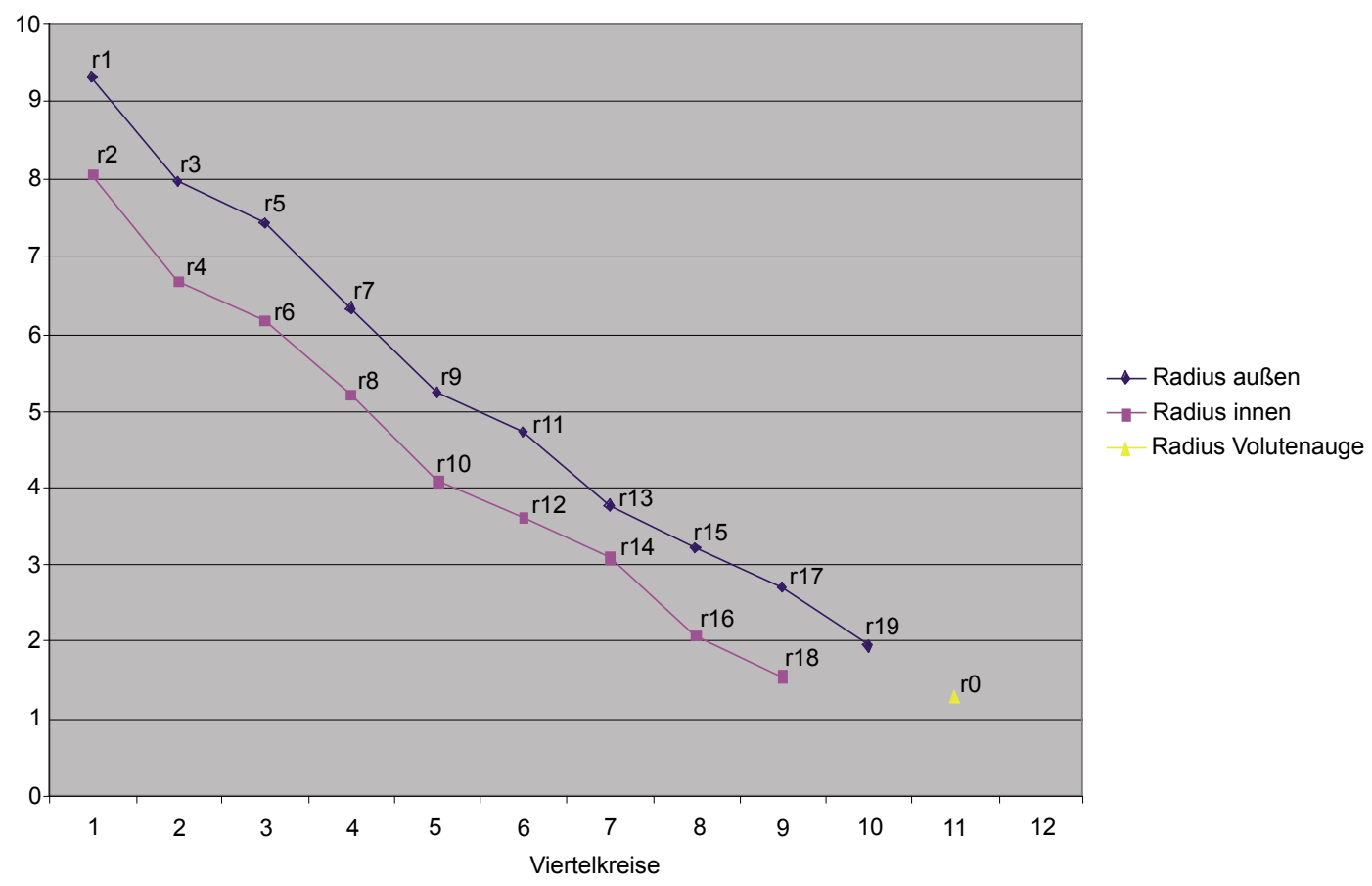

Tabelle 5: Canalis-Breite Volute 2

\begin{tabular}{|c|c|c|}
\hline Schnittpunkt & Breite Canalis & moduli \\
\hline 1 & $2,72 \mathrm{~cm}$ & $2 \frac{1}{8}$ \\
\hline 2 & $2,43 \mathrm{~cm}$ & $1^{\frac{1}{10}}$ \\
\hline 3 & $2,16 \mathrm{~cm}$ & $1 \frac{1}{3}$ \\
\hline 4 & $1,76 \mathrm{~cm}$ & $1 \frac{3}{8}$ \\
\hline 5 & $1,62 \mathrm{~cm}$ & $1 \frac{1 / 4}{11 / 8}$ \\
\hline 6 & $1,43 \mathrm{~cm}$ & $1 \frac{1 / 8}{7 / 8}$ \\
\hline 7 & $1,50 \mathrm{~cm}$ & $4 / 10$ \\
\hline 8 & $1,12 \mathrm{~cm}$ & \\
\hline 9 & $0,55 \mathrm{~cm}$ & \\
\hline
\end{tabular}

Die Proportionalverhältnisse der Volute stimmen nicht mit den Vorgaben Vitruvs überein: So verhält sich die Höhe der Volute über dem Zentrum des Volutenauges zur Höhe darunter nicht wie die vorgegebenen $9 \mathrm{r} 0: 7 \mathrm{r} 0$, sondern wie 73/4 $\mathrm{r} 0\left(9,99 \mathrm{~cm}=5 \frac{1}{2}\right.$ digiti $): 61 / 4 \mathrm{r} 0\left(7,94 \mathrm{~cm}=4 \frac{1}{4}\right.$ digiti $)$, kurz $73 / 4: 61 / 4$. Die Gesamthöhe der Volute entspricht nicht den vorgegebenen 8 Durchmessern des Volutenauges (16 r0), sondern lediglich 14 r0 (17,93 cm =93/4 digiti). Die Abnahme der Radien der ersten vier Viertelkreise um den Radius des Volutenauges wird nicht eingehalten: 71/4-61/4-53/4-5. Das maximale Breitenverhältnis der Volute lautet $7 \mathrm{r} 0(8,94 \mathrm{~cm}=43 / 4$ digiti $): 5 \mathrm{r} 0\left(6,52 \mathrm{~cm}=3 \frac{1}{2}\right.$ digiti $)$. Für das Verhältnis Höhe : Breite der Volute ergibt dies $14 \mathrm{r} 0(17,93 \mathrm{~cm}=93 / 4$ digiti $): 12 \mathrm{r} 0\left(15,46 \mathrm{~cm}=8 \frac{1}{3}\right.$ digiti $)$, d. h. $3 \frac{1}{2}: 3$. 


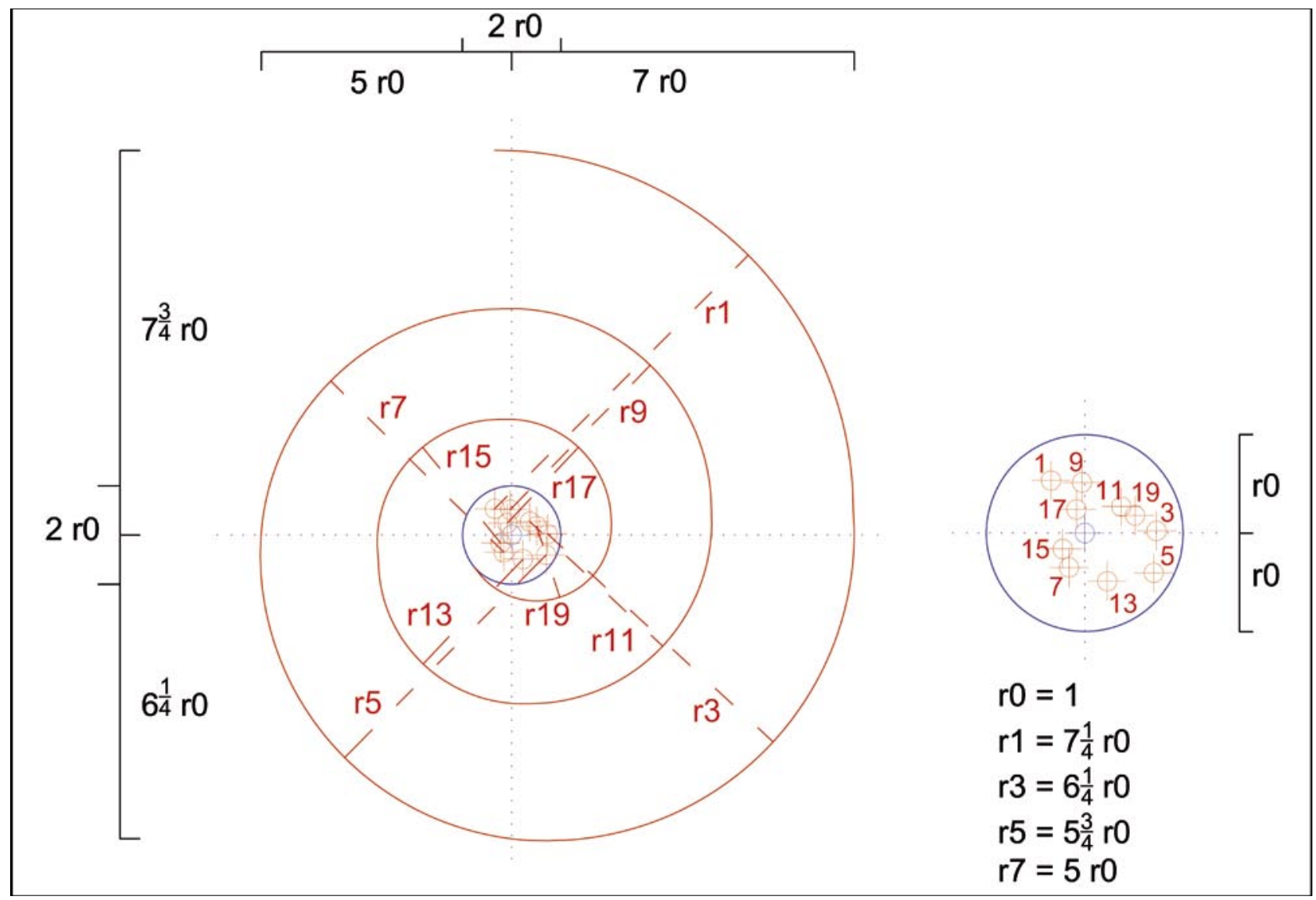

12 Werkriss Volute 2 - Detail äußere Windung M. $1: 2$ (Volutenauge M. $1: 1$ )

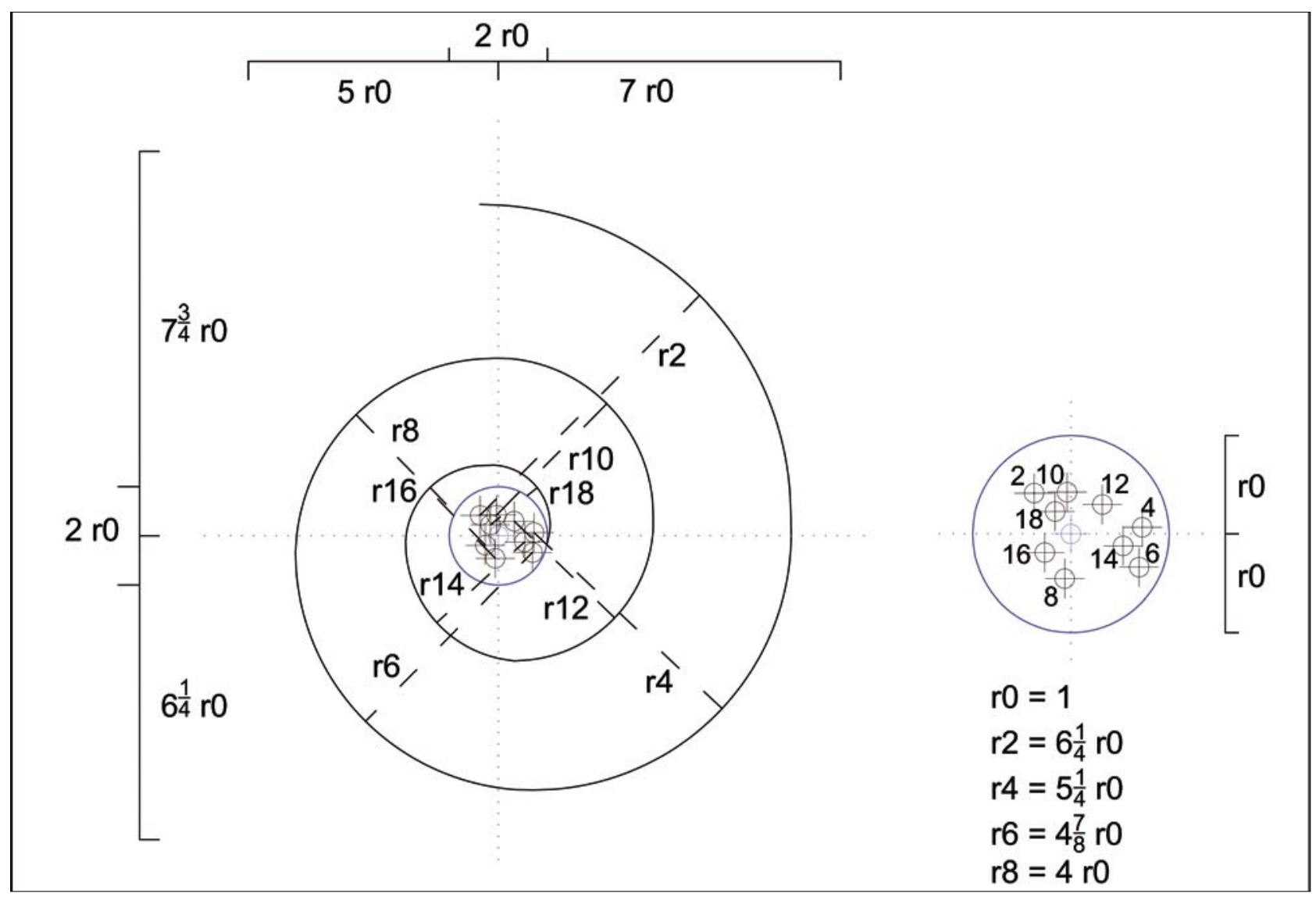

13 Werkriss Volute 2 - Detail innere Windung M. $1: 2$ (Volutenauge M. $1: 1$ ) 
Canalis-Breite Volute 2

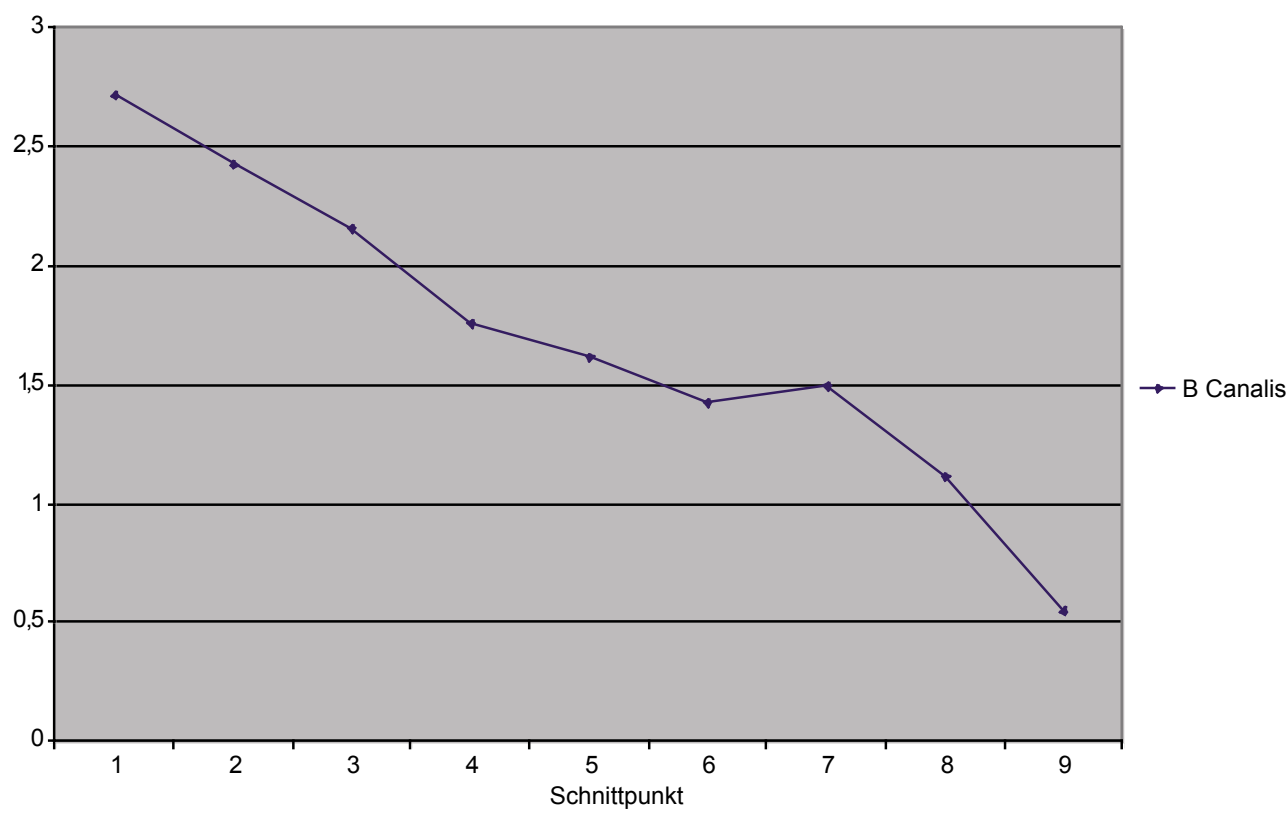

Tabelle 6: Proportionsverhältnisse Volute 2

\begin{tabular}{|c|c|c|c|}
\hline & $\begin{array}{c}\text { Höhenproportion an } \\
\text { Horizontalachse }\end{array}$ & $\begin{array}{c}\text { Breitenproportion an } \\
\text { Vertikalachse }\end{array}$ & $\begin{array}{c}\text { Proportion } \\
\text { Höhe : Breite }\end{array}$ \\
\hline Volute 2 & $73 / 4: 61 / 4$ & $7: 5$ & $31 / 2: 3$ \\
\hline
\end{tabular}

Analog der von H. Büsing vorgenommenen Analyse zu den Voluten der Kapitelle der ErechtheionOsthalle entspricht auch bei den vorgeritzten Voluten im Prytaneion die Summe aller Wegstrecken zwischen den Zirkelpunkten dem Radius des jeweiligen ersten Viertelkreises ${ }^{32}$. Dieses geometrische Axiom ist auf alle griechischen und römischen Kapitelle mit ionischen Voluten anwendbar. Die Summe der Wegstrecken beginnt mit der Distanz des Mittelpunktes des Volutenauges zum Zentrum des ersten Viertelkreises und endet nach Addition der Distanzen zwischen den Mittelpunkten der Viertelkreise wiederum im Zentrum des Volutenauges.

Tabelle 7: Summe der Wegstrecken und Radien der ersten Viertelkreise

\begin{tabular}{|c|c|c|}
\hline Volute & $\begin{array}{c}\text { Summe der Wegstrecken } \\
\text { zwischen den Zirkelpunkten }\end{array}$ & Radius 1. Viertelkreis \\
\hline Volute 1, äußere Windung & $13,88 \mathrm{~cm}$ & $13,88 \mathrm{~cm}(\mathrm{r} 1)$ \\
\hline Volute 1, innere Windung & $11,78 \mathrm{~cm}$ & $11,78 \mathrm{~cm} \mathrm{(r2)}$ \\
\hline Volute 2, äußere Windung & $9,31 \mathrm{~cm}$ & $9,31 \mathrm{~cm}(\mathrm{r} 1)$ \\
\hline Volute 2, innere Windung & $8,08 \mathrm{~cm}$ & $8,08 \mathrm{~cm}(\mathrm{r} 2)$ \\
\hline
\end{tabular}

\footnotetext{
${ }^{32}$ Vgl. Büsing - Lehnhoff 1985, 106-119.
} 


\section{Weitere Konstruktionslinien}

Die zahlreichen weiteren auf dem Säulenpostament befindlichen Ritzlinien sind nur mit Vorbehalt als Werkrisse zu interpretieren und stehen, abgesehen von der horizontalen Konstruktionsachse, die fast über die gesamte Südseite des Postaments läuft (L 92,94 cm), in keinem funktionalem Zusammenhang mit der Ritzzeichnung der Voluten.

Von Interesse sind aber die auf einer Länge von ca. $38 \mathrm{~cm}$ zwischen den Voluten zu erkennenden, zumindest vier annähernd konzentrischen Kreisbögen mit Radien von ca. 80,68, 79,69, 77,87 und 77,77 cm, welche die beiden Voluten gleichsam als Übergangsbögen verbinden.

\section{Synthese}

Die Analyse des Werkrisses führt zu folgenden Ergebnissen: Die Geometrie der beiden vorgestellten Voluten entspricht nur teilweise dem starren, bei Vitruv beschriebenen Konstruktionsschema. Die Voluten sind vielmehr frei geformt, der Verlauf ihrer Windungen eher organisch-homogen als geometrisch-regelmäßig. Die unregelmäßige Anordnung der Einstichpunkte zeugt von dieser freien Gestaltungsweise ${ }^{33}$.

Da die Viertelkreise der Voluten nicht immer konzentrisch verlaufen und somit bei Verwendung eines Volutenzirkels sehr viele Nägel in einem recht klein dimensionierten Volutenauge anzubringen gewesen wären, erfolgte die Übertragung der Zeichnung auf das Werkstück wahrscheinlich mithilfe einer Lochschablone ${ }^{34}$.

Es wäre nun reizvoll, den Werkriss einem im Gebäude tatsächlich verbauten Bauglied zuweisen zu können. Da im Prytaneion von Ephesos dafür nur die Kompositkapitelle im Hestiasaal - es wurden hier bis dato keine weiteren Architekturglieder mit Voluten, wie etwa von der ionischen Ordnung des Vorhofes, gefunden - in Frage kommen, galt es, dies zu überprüfen. Dies umso mehr, als die Voluten dieser Kapitelle ebenfalls $2 \frac{1}{2}$ Windungen besitzen. Die Abmessungen des am besten erhaltenen Kompositkapitells auf der nordwestlichen Herzsäule (Inv. PR 23/07) gestalten sich wie folgt (Abb. 14):

Kapitell Inv. PR 23/07

$\begin{array}{ll}\text { H } & 74 \mathrm{~cm} \\ \text { L und B Kapitellbasis } & 80 \times 80 \mathrm{~cm} \\ \text { H Abakus } & 13 \mathrm{~cm} \\ \text { B Abakus } & 104 \times 105 \mathrm{~cm} \\ \text { H Echinus } & 11,5 \mathrm{~cm} \\ \text { B Kalathoslippe } & 2,5 \mathrm{~cm} \\ \text { H Kalathos gesamt } & 49,5 \mathrm{~cm} \\ \text { H Blattstab } & 13 \mathrm{~cm} \\ \text { H Hochblatt } & 29 \mathrm{~cm} \\ \text { H Lanzettblatt } & 29 \mathrm{~cm} \\ \text { H Volute } & 17 \mathrm{~cm} \\ \text { B Volute } & 16 \mathrm{~cm}\end{array}$

Die Voluten der Kompositkapitelle der Herzsäulen sind somit deutlich kleiner als Volute 1 bzw. etwas gedrungener proportioniert als Volute 2. Die Konstruktionszeichnung spielte bei der Herstellung dieser Kapitelle folglich keine Rolle.

\footnotetext{
${ }^{33}$ Die Konstruktionszeichnung dürfte von einem geübten Anreißer ausgeführt worden sein, der sich grob an der bei Vitruv beschriebenen Vorgehensweise orientierte, die Zirkelpunkte auf der vorgestellten Zeichnung aber teilweise frei - aus seiner Erfahrung und Praxis heraus - setzte.

${ }^{34}$ s. dazu Heisel (Anm. 11) 214-216; Loertscher 1989, 84.
} 
Tabelle 7: Gegenüberstellung der Proportionen

\begin{tabular}{|c|c|l|l|c|}
\hline Volute & Anzahl Windungen & Höhe & Breite & Höhe : Breite \\
\hline Kompositkapitell (Inv. PR 23/07) & $2^{1 / 2}$ & $17 \mathrm{~cm}$ & $16 \mathrm{~cm}$ & $4 \frac{1}{2}: 4$ \\
\hline Volute 1 & $2^{1 / 2}$ & $26,27 \mathrm{~cm}$ & $22,99 \mathrm{~cm}$ & $4: 3^{1 / 2}$ \\
\hline Volute 2 & $2^{1 / 2}$ & $17,93 \mathrm{~cm}$ & $15,46 \mathrm{~cm}$ & $3 \frac{1}{2}: 3$ \\
\hline
\end{tabular}

Betrachtet man aber die beiden Voluten, die ja an einer gemeinsamen Horizontalachse ausgerichtet sind, als einen zusammengehörigen Werkriss, wie es die als Übergangsbögen ausgebildeten Ritzlinien zwischen den Voluten implizieren, so zeichnen sich zwei S-förmig angeordnete Voluten ab, wobei die größere Volute 1 als stehende und die kleinere Volute 2 als hängende Volute konstruiert ist. Eine große Voluteneinrollung am Ansatz und eine kleine vorn entsprechen mit Voluten ausgearbeiteten Türkonsolen ${ }^{35}$, dem seitlichen Schmuck von Geisa mit Volutenkonsolen, wie sie ab frühaugusteischer Zeit nachweisbar sind ${ }^{36}$, Volutenakroteren $^{37}$ sowie S-Spiralvolutenkapitellen ${ }^{38}$. Durch die verschiedenen Größen der Voluten bildeten sich auf der Unterseite auch zwei unterschiedlich große Polster.

Bei dem Werkriss auf dem südwestlichen Säulenpostament im Hestiasaal des ephesischen Prytaneions handelt es sich folglich mit großer Sicherheit um die 78,31 cm (42 $\frac{1}{3}$ digiti) tiefe Seitenfläche eines Konsolengeisons, um eine mit Voluten verzierte Türkonsole oder die Doppelvolute eines Volutenakroters bzw. eines S-Spiralvolutenkapitells. Während Volute 1 sich an von Vitruv vorgegebenen Proportionsverhältnissen - insbesondere am modulus des Volutenaugen-Radius - orientiert, jedoch auch in einigen Bereichen von ihnen abweicht, scheint Volute 2 einem völlig anderen

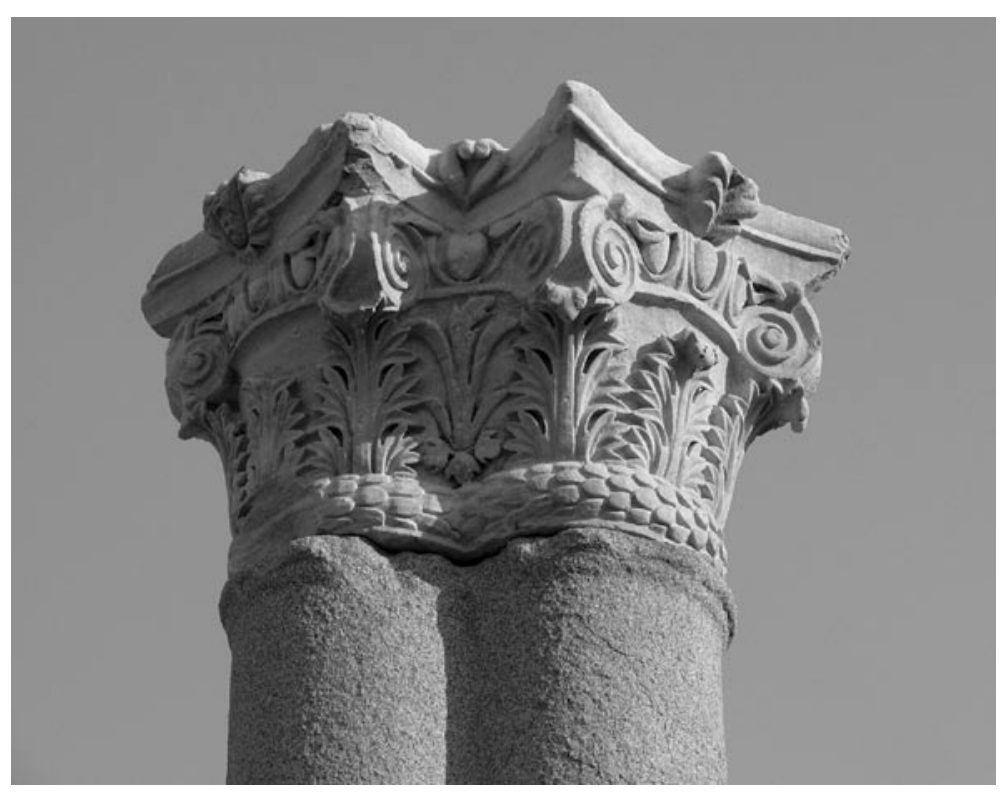

14 Prytaneion - Hestiasaal. >Herzkapitell< der Nordwestssäule

${ }^{35}$ vgl. A. Büsing-Kolbe, Frühe griechische Türen, JdI 93, 1978, 66-174; H. Klenk, Die antike Tür (Gießen 1924) 3 f.; F. Rumscheid, Untersuchungen zur kleinasiatischen Bauornamentik des Hellenismus, BeitrESkAr 14 (Mainz 1994) 330 f.; Vitr. 4, 6,4 .

${ }^{36}$ s. dazu grundlegend: H. v. Hesberg, Konsolengeisa des Hellenismus und der frühen Kaiserzeit, RM Ergh. 24 (Mainz 1980) 205-217.

${ }^{37}$ In Ephesos fanden sich Volutenakrotere beispielsweise am Theater, am Nymphaeum Traiani sowie am Heraklestor (?); vgl. A. Öztürk, Die Restaurierung des Bühnengebäudes des Theaters von Ephesos, ÖJh 75, 2006, 212 Abb. 12 (Theater); U. Quatember, Das Nymphaeum Traiani in Ephesos, FiE 11, 2 (in Druck); A. Bammer, Ein spätantiker Torbau aus Ephesos, ÖJh 51, 1976/1977, Beibl. 121 f. Abb. 29 (Heraklestor). Im Hanghaus 2, Wohneinheit 6, Raum 31b war eine Ädikula von zwei Doppelvoluten bekrönt; vgl. U. Quatember, Private Kulteinrichtungen im Hanghaus 2 in Ephesos, in: B. Asamer - W. Wohlmayr (Hrsg.), Akten des 9. Österreichischen Archäologentages am Institut für Klassische Archäologie der Paris-Lodron-Universität Salzburg 6.-8. Dezember 2001 (Wien 2003) 173 f. - Keine dieser Voluten besitzt die gleichen Maße wie die vorgeritzten Voluten im Prytaneion oder ein Vielfaches von ihnen. Dies trifft auch auf die übrigen im Gelände verstreut anzutreffenden Volutenakrotere zu. Für Hinweise zu den Stücken am Nymphaeum Traiani, Heraklestor (?) und Hanghaus 2 sei U. Quatember gedankt.

${ }^{38}$ s. dazu M. Koller, Die Pilasterkapitelle aus dem ^Marmorsaal`, in: F. Krinzinger (Hrsg.), Das Hanghaus 2 von Ephesos. Studien zu Baugeschichte und Chronologie, AForsch 7 = DenkschrWien 302 (Wien 2002) 119-136. Bei den Beispielen im Hanghaus 2 enden die unteren Voluten aber großteils vegetabil oder in Knöpfen und entsprechen daher nicht dem Konstruktionsschema des Werkrisses im Prytaneion. Beispiele von S-Spiralvolutenkapitellen mit voll ausgebildeten - allerdings anders proportionierten - Voluten finden sich am Bühnengebäude des Theaters von Ephesos. Zwei dieser Kapitelle werden im KHM Wien verwahrt (KHM ANSA I 1631e. I 1631d); vgl. A. Bammer in: W. Oberleitner, Funde aus Ephesos und Samothrake, Katalog der Antikensammlung 2 (Wien 1978) 65 Nr. 51. 52; Plattner (Anm. 3) 229. 
konstruktiven Kanon zu entspringen, der aber ebenso dem modulus des Volutenaugen-Radius folgt. Dies spiegelt sich besonders deutlich im klaren Verhältnis von Höhe zu Breite wider $\left(3 \frac{1}{2}: 3\right)$.

Handelte es sich bei der Zeichnung um die Voluten eines Konsolengeisons, eines S-Spiralvolutenkapitells oder eines Volutenakroters, so kann ein Konnex mit dem Gebäude ausgeschlossen werden, da im Prytaneion solche Architekturglieder zu keiner Zeit verbaut waren. Wo ein solches Konsolengeison in Ephesos tatsächlich versetzt gewesen sein könnte, ist vorderhand nicht zu klären, zumal eine entsprechende Konsole mit einer derart großen Tiefe bisher nicht gefunden wurde und auch keinem bekannten Gebäude zugewiesen werden kann $^{39}$. Es dürfte sich bei dem vorliegenden Werkriss somit um die Doppelvolute eines Volutenakroters, eines S-Spiralvolutenkapitells oder die Voluten einer Türkonsole handeln, genauer gesagt, um die Volutenglieder eines ionischen Türrahmens, die an beiden Seiten den Türsturz und das Hyperthyron begrenzten ${ }^{40}$. Über die Verzierung der Doppelvolute kann in all diesen Fällen nichts gesagt werden. Auf Basis der Konstruktionszeichnung ist auch die Anbringung des Volutenakroters oder die Verbindung der Volutenkonsole mit der Türverdachung nicht zu klären. Als Datierungskriterium für die Herstellung des Werkrisses kann daher nur ein terminus post quem in augusteischer Zeit, sprich der Errichtungszeit des Prytaneions, festgesetzt werden.

Lediglich die Türkonsole könnte tatsächlich im Prytaneion baulich umgesetzt gewesen sein, doch ist dies wegen des Erhaltungszustandes des Monuments nicht gesichert. Sollte der Werkriss auch praktischen Sinn besessen haben, wovon auszugehen ist, dürfte der Anbringungsort der Konsolen, des Akroters oder des Kapitells aber nicht allzu weit von ihm entfernt gewesen sein. Nur so wäre ein sinnvolles und ergonomisches Abgreifen und Ausarbeiten der Voluten gewährleistet gewesen.

Mag. Dr. Martin Steskal

Österreichisches Archäologisches Institut, Franz Klein-Gasse 1, A-1190 Wien

E-Mail: martin.steskal@oeai.at

Abbildungsnachweis: Abb. 1. 2: Photo M. Steskal, (C ÖAI; Abb. 3: Graphik J. Eitler, L. Zabrana, (C ÖAI; Abb. 4: Graphik M. Steskal nach Büsing 1987, Abb. 1; Abb. 5: Graphik N. High, M. Steskal, L. Zabrana, (C) ÖAI; Abb. 6. 10: Photo N. Gail, (C) ÖAI; Abb. 7-9. 11-13: Graphik M. Steskal; Abb. 14: Photo M. Steskal, (C) ÖAI.

${ }^{39}$ Ch. Baier, Kaiserzeitliche Konsolengeisa in Kleinasien. Untersuchungen zur Bauornamentik von flavischer bis in severische Zeit am Beispiel Ephesos (Mag. Universität Wien 2006).

${ }^{40}$ Zur ionischen Tür: Büsing-Kolbe (Anm. 35) 82-142. - Nur wenige solcher ephesischer Volutenkonsolen sind tatsächlich bekannt. Von folgenden Bauten haben sich Beispiele erhalten: ১Hadrianstempek (in situ), Celsusbibliothek (in situ), Serapeion, Mittleres Hafentor (heute Wien: KHM ANSA I 1629a) und Theater. Darüber hinaus werden im KHM Wien folgende Streufunde verwahrt: Fundort Ephesos (KHM ANSA I 1638), Fundort Bereich des Theaters (KHM ANSA I 1631g). Keine dieser Voluten besitzt die gleichen Maße wie die vorgeritzten Voluten im Prytaneion oder ein Vielfaches von ihnen. Für Hinweise zu den Funden im KHM Wien und dem Neufund im Theater sei G. A. Plattner gedankt. 